\title{
Complexity of suffix-free regular languages
}

\author{
Janusz A. Brzozowski ${ }^{a}$, Marek Szykuła ${ }^{b}$ \\ ${ }^{a}$ David R. Cheriton School of Computer Science, University of Waterloo, \\ Waterloo, ON, Canada N2L $3 G 1$ \\ ${ }^{b}$ Institute of Computer Science, University of Wrocław, Joliot-Curie 15, \\ PL-50-383 Wrocław, Poland
}

\begin{abstract}
We study various complexity properties of suffix-free regular languages. A sequence $\left(L_{k}, L_{k+1}, \ldots\right)$ of regular languages in some class, where $n$ is the quotient complexity of $L_{n}$, is most complex if its languages $L_{n}$ meet the complexity upper bounds for all basic measures. It is known that there exist such most complex sequences in several classes of regular languages. In contrast to this, we prove that there does not exist a most complex sequence in the class of suffix-free regular languages. However, we do exhibit two such sequences that together meet upper bounds for all basic measures.

Keywords: most complex, regular language, state complexity, suffix-free, syntactic complexity, transition semigroup
\end{abstract}

\section{Introduction}

We study complexity properties of suffix-free regular languages. A much shorter preliminary version of these results appeared in [19] without any proofs.

Motivation The state complexity $\kappa(L)$ of a regular language $L$ over an alphabet $\Sigma$ is the number of states in a minimal deterministic finite automaton (DFA) with input alphabet $\Sigma$ recognizing $L$. The state complexity of a regularity preserving unary operation $\circ$ on regular languages is the maximal value of $\kappa\left(L_{n}^{\circ}\right)$ as a function of $n$, where $L_{n}$ varies over all regular languages $L_{n}$ with state complexity $n$. Similarly, the state complexity of a regularity preserving binary operation $\circ$ on regular languages is the maximal value of $\kappa\left(K_{m} \circ L_{n}\right)$ as a function of $m$ and $n$, where $K_{m}$ and $L_{n}$ vary over all regular languages of state complexities $m$ and $n$, respectively. Of special

Email addresses: brzozo@uwaterloo.ca (Janusz A. Brzozowski), msz@cs.uni.wroc.pl (Marek Szykuła) 
interest are the state complexities of common operations on regular languages. The state complexities of union, product (concatenation), (Kleene) star and reversal were studied by Maslov [33] in 1970, but this work was not well known for many years. The paper by Yu, Zhuang, and Salomaa [37] inspired considerable interest in these problems and much research has been done on this topic in the past 22 years. The state complexity of an operation gives a worst-case lower bound on the time and space complexities of the operation. For this reason it has been studied extensively; see $[5,28,36]$ for additional references.

Consider, for example, the product of two regular languages. To find its state complexity we need to establish an upper bound for it, and find two languages of state complexities $m$ and $n$, respectively that meet this bound. It is known that $(m-2) 2^{n}+2^{n-1}$ is a tight upper bound on product. The languages that meet this bound are called witnesses. In general, different witnesses have been used for the two arguments and for different operations. However, Brzozowski [6] has shown that one witness and its slightly modified version called a dialect is sufficient to meet the bounds on all binary Boolean operations, product, star, and reversal. The DFA of this witness is shown in Figure 1. Let the language recognized by this DFA be $L_{n}(a, b, c)$, and let $L_{n}(b, a, c)$ be the language of the DFA obtained from that of Figure 1 by interchanging the roles of $a$ and $b$. Then $L_{m}(a, b, c)$ and $L_{n}(b, a, c)$ meet the bound $m n$ for all binary boolean operations, $L_{m}(a, b, c)$ and $L_{n}(a, b, c)$ meet the bound $(m-1) 2^{n}+2^{n-1}$ for product, and $L_{n}(a, b, c)$ meets the bounds $2^{n-1}+2^{n-2}$ for star and $2^{n}$ for reversal.

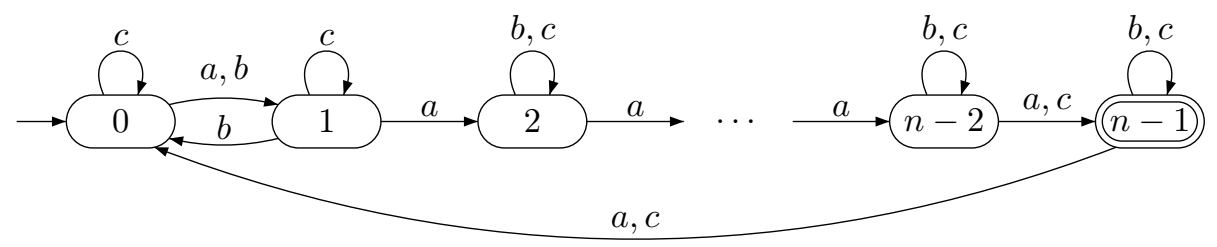

Figure 1: Minimal DFA of a most complex regular language.

Although state complexity is a useful measure, it has some deficiencies. The language $L_{n}(a, b, c)$ and the language $\{a, b, c\}^{n-2}$ both have state complexity $n$, but the second language is intuitively much simpler. Secondly, all the bounds for common operations - except reversal, which misses the bound by one state - are also met by star-free languages [13], where the class of star-free languages is the smallest class containing the finite languages and closed under Boolean operations and product, but not star. For these reasons Brzozowski [6] suggested additional complexity mea- 
sures: the maximal size of the syntactic semigroup of the language and the state complexities of atoms (discussed later). It turns out that the language defined in Figure 1 also meets these bounds. For these reasons this language has been called a most complex regular language. The added measure of the size of the syntactic semigroup distinguishes well between $L_{n}(a, b, c)$ and $\{a, b, c\}^{n-1}$ since the semigroup of the first language has $n^{n}$ elements, while the second has $n-1$ elements.

In previous work on state complexity it was always assumed that the two arguments in product and binary Boolean operations are restricted to be over the same alphabet. But Brzozowski pointed out in 2016 [7] that operations on languages over different alphabets, unrestricted operations, have higher tight upper bounds. It was shown that witnesses very similar to those of Figure 1 also meet these bounds $[7,16]$, and thus are most complex for both restricted and unrestricted operations. For suffix-free languages the restricted and unrestricted complexities are the same.

Most complex languages are useful for testing the efficiency of systems. Hence to check the maximal size of the objects that a system can handle, we can use most complex languages. It is certainly simpler to have just one or two worst-case examples.

The question now arises whether most complex languages also exist in subclasses of regular languages. A natural source of subclasses is obtained from the notion of convexity. Convex languages were introduced by Thierrin [35] and studied later in [1]. They can be defined with respect to arbitrary binary relations on an alphabet $\Sigma^{*}$, but the relations "is a prefix of", "is a suffix of" and "is a factor of" turned out to be of considerable interest, where if $w=x y z \in \Sigma^{*}$, then $x$ is a prefix of $w, y$ is a factor, and $z$ is a suffix. A language $L$ is prefix-convex if, whenever $w=x y z$ and $w$ and $x$ are in $L$, then so is xy. Factor-convex and suffix-convex languages are defined in a similar way. Brzozowski began studying the complexity properties of these languages in [4].

The class of prefix-convex languages has four natural subclasses: right ideals (languages $L$ satisfying $L=L \Sigma^{*}$ ), prefix-closed languages (where $w$ in $L$ implies that every prefix of $w$ is in $L$ ), prefix-free languages (where $w$ in $L$ implies that no prefix of $w$ other than $w$ is in $L$ ), and prefix-proper languages that are prefix-convex but do not belong to any one of the three special subclasses. Similarly, there are four subclasses of suffix-free languages: left ideals (languages $L$ satisfying $L=\Sigma^{*} L$ ), suffix-closed languages, suffix-free languages, and suffix-proper languages. Finally, there are four subclasses of factor-free languages: two-sided ideals (languages $L$ satisfying $L=$ $\left.\Sigma^{*} L \Sigma^{*}\right)$, factor-closed languages, factor-free languages, and factor-proper languages. Ideals appear in pattern matching [24]: if we are looking for all the words in a given text that begin with words in a pattern language $L$, then we are dealing 
with the right ideal $L \Sigma^{*}$, and similar statements apply to left and two-sided ideals. Prefix-closed (respectively, suffix-closed, factor closed) languages are complements of right ideals (respectively, left ideals, two-sided ideals). Prefix-free (respectively, suffix-free, factor-free) languages, other than the language consisting of the empty word, are codes [3], and have many applications, particularly in cryptography, data compression and error correction.

Most complex left, right and two-sided ideals for restricted operations were found in [10], and unrestricted operations were added in [16]. Most complex prefix-closed, and prefix-free languages were exhibited in [17] and proper prefix-convex languages in [15]. Most complex suffix-closed languages were found in [14]. In contrast to these results, the first example of a subclass of the class of regular languages that does not have a most complex language is that of suffix-free languages. This result was reported in [20], and is the subject of the present paper. It is also known (unpublished result) that most complex proper suffix-convex languages do not exist.

Quotient Complexity A basic complexity measure of a regular language $L$ over an alphabet $\Sigma$ is the number $n$ of distinct left quotients of $L$, where a (left) quotient of $L$ by a word $w \in \Sigma^{*}$ is $w^{-1} L=\{x \mid w x \in L\}$. We denote the set of quotients of $L$ by $K=\left\{K_{0}, \ldots, K_{n-1}\right\}$, where $K_{0}=L=\varepsilon^{-1} L$ by convention. Each quotient $K_{i}$ can be represented also as $w_{i}^{-1} L$, where $w_{i} \in \Sigma^{*}$ is such that $w_{i}^{-1} L=K_{i}$. The number of quotients of $L$ is its quotient complexity [5]. Since the set of quotients of $L$ is the same as the number of states in a minimal DFA recognizing $L$, the quotient complexity of $L$ is the same as its state complexity; however, quotient complexity suggests languagetheoretic methods, whereas state complexity deals with automata. The quotient complexities of unary operation and binary operations are defined analogously to state complexities.

To establish the state/quotient complexity of a regularity preserving unary operation $\circ$ we need a sequence $\left(L_{n}, n \geqslant k\right)=\left(L_{k}, L_{k+1}, \ldots\right)$, called a stream, of witness languages that meet this bound; here $k$ is usually some small integer because the bound may not apply for $n<k$. In all the cases that have been studied the languages in a stream are defined in the same way, differing only in the parameter $n$. For example, the languages of the DFAs of Figure 1 define the stream $\left(L_{n}(a, b, c) \mid n \geqslant 3\right)$.

To establish the state/quotient complexity of a regularity preserving binary operation $\circ$ on regular languages, for each pair $(m, n), m \geqslant h, n \geqslant k$, we need to find two languages $K_{m, n}$ and $L_{m, n}$ meeting this bound; here $K_{m, n}$ and $L_{m, n}$ are of complexities $m$ and $n$, respectively. The notation $K_{m, n}$ and $L_{m, n}$ implies that $K_{m, n}$ depends on $n$, and $L_{m, n}$ depends on $m$. If $K_{m, n}$ together with $L_{m, n}$ meet the required bound, it may occur that $K_{m, n}$ with $L_{m^{\prime}, n}$ may not meet that bound. Indeed, two such examples have been found by Han and Salomaa [25]: the union and intersec- 
tion of finite languages require such witnesses. Fortunately, however, in all other cases studied in the literature, it suffices to use witness streams $\left(K_{m}, m \geqslant h\right)$ and $\left(L_{n}, n \geqslant k\right)$, where $K_{m}$ is independent of $n$ and $L_{n}$ is independent of $m$. This is the case for suffix-free languages. The state/quotient complexity of suffix-free languages using various witnesses was examined in [23, 26, 30].

We also extend the notions of maximal complexity, stream, and witness to DFAs. Syntactic Complexity A second measure of complexity of a regular language is its syntactic complexity. Let $\Sigma^{+}$be the set of non-empty words of $\Sigma^{*}$. The syntactic semigroup of $L$ is the set of equivalence classes of the Myhill congruence $\approx_{L}$ on $\Sigma^{+}$defined by $x \approx_{L} y$ if and only if $u x v \in L \Leftrightarrow u y v \in L$ for all $u, v \in \Sigma^{*}$. The syntactic semigroup of $L$ is isomorphic to the transition semigroup of a minimal DFA $\mathcal{D}$ recognizing $L$ [34], which is the semigroup of transformations of the state set of $\mathcal{D}$ induced by non-empty words. The syntactic complexity of $L$ is the cardinality of its syntactic/transition semigroup.

Holzer and König [27], and independently Krawetz, Lawrence and Shallit [31] studied the syntactic complexity in the classes of unary and binary regular languages. This problem was also solved for the classes of right ideals [16, 22], left ideals [16, 18, 22], two-sided ideals [16, 18, 22], prefix-free languages [12], and suffixfree languages $[12,19]$.

Complexities of Atoms A possible third measure of complexity of a regular language $L$ is the number and quotient complexities, which we call simply complexities, of certain languages, called atoms, uniquely defined by $L$. Atoms arise from an equivalence on $\Sigma^{*}$ which is a left congruence refined by the Myhill congruence, where two words $x$ and $y$ are equivalent if $u x \in L$ if and only if $u y \in L$ for all $u \in \Sigma^{*}$ [29]. Thus $x$ and $y$ are equivalent if $x \in u^{-1} L \Leftrightarrow y \in u^{-1} L$. An equivalence class of this relation is called an atom [21] of $L$. It follows that an atom is a non-empty intersection of complemented and uncomplemented quotients of $L$. The quotients of a language are unions of its atoms.

Terminology and Notation A deterministic finite automaton (DFA) is defined as a quintuple $\mathcal{D}=\left(Q, \Sigma, \delta, q_{0}, F\right)$, where $Q$ is a finite non-empty set of states, $\Sigma$ is a finite non-empty alphabet, $\delta: Q \times \Sigma \rightarrow Q$ is the transition function, $q_{0} \in Q$ is the initial state, and $F \subseteq Q$ is the set of final states. We extend $\delta$ to a function $\delta: Q \times \Sigma^{*} \rightarrow Q$ as usual. A DFA $\mathcal{D}$ accepts a word $w \in \Sigma^{*}$ if $\delta\left(q_{0}, w\right) \in F$. The language accepted by $\mathcal{D}$ is denoted by $L(\mathcal{D})$. If $q$ is a state of $\mathcal{D}$, then the language $L^{q}$ of $q$ is the language accepted by the DFA $(Q, \Sigma, \delta, q, F)$. A state is empty if its language is empty. Two states $p$ and $q$ of $\mathcal{D}$ are equivalent if $L^{p}=L^{q}$. A state $q$ is reachable if there exists $w \in \Sigma^{*}$ such that $\delta\left(q_{0}, w\right)=q$. A DFA is minimal if all of its states are reachable and no two states are equivalent. Usually DFAs are 
used to establish upper bounds on the quotient complexity of operations and also as witnesses that meet these bounds.

A nondeterministic finite automaton (NFA) is a quintuple $\mathcal{D}=(Q, \Sigma, \delta, I, F)$, where $Q, \Sigma$ and $F$ are defined as in a DFA, $\delta: Q \times \Sigma \rightarrow 2^{Q}$ is the transition function, and $I \subseteq Q$ is the set of initial states. An $\varepsilon-N F A$ is an NFA in which transitions under the empty word $\varepsilon$ are also permitted.

The quotient $D F A$ of a regular language $L$ with $n$ quotients is defined by $\mathcal{D}=$ $\left(K, \Sigma, \delta_{\mathcal{D}}, K_{0}, F_{\mathcal{D}}\right)$, where $\delta_{\mathcal{D}}\left(K_{i}, w\right)=K_{j}$ if and only if $w^{-1} K_{i}=K_{j}$, and $F_{\mathcal{D}}=$ $\left\{K_{i} \mid \varepsilon \in K_{i}\right\}$. To simplify the notation, without loss of generality we use the set $Q_{n}=\{0, \ldots, n-1\}$ of subscripts of quotients as the set of states of $\mathcal{D}$; then $\mathcal{D}$ is denoted by $\mathcal{D}=\left(Q_{n}, \Sigma, \delta, 0, F\right)$, where $\delta(p, w)=q$ if $\delta_{\mathcal{D}}\left(K_{p}, w\right)=K_{q}$, and $F$ is the set of subscripts of quotients in $F_{\mathcal{D}}$. The quotient DFA of $L$ is unique and it is isomorphic to each complete minimal DFA of $L$.

A transformation of $Q_{n}$ is a mapping $t: Q_{n} \rightarrow Q_{n}$. The image of $q \in Q_{n}$ under $t$ is denoted by $q t$. The range of $t$ is $\operatorname{rng}(t)=\left\{q \in Q_{n} \mid p t=q\right.$ for some $\left.p \in Q_{n}\right\}$. In any DFA, each letter $a \in \Sigma$ induces a transformation $\delta_{a}$ of the set $Q_{n}$ defined by $q \delta_{a}=$ $\delta(q, a)$. By a slight abuse of notation we use the letter $a$ to denote the transformation it induces; thus we write $q a$ instead of $q \delta_{a}$. We also extend the notation to sets of states: if $P \subseteq Q_{n}$, then $P a=\{p a \mid p \in P\}$. If $s, t$ are transformations of $Q$, their composition is denoted by $s * t$ and defined by $q(s * t)=(q s) t$; the $*$ is usually omitted. Then also for a word $w=a_{1} \cdots a_{k}, \delta_{w}$ denotes the transformation $\delta_{a_{1}} \cdots \delta_{a_{k}}$ induced by $w$. Let $\mathcal{T}_{Q_{n}}$ be the set of all $n^{n}$ transformations of $Q_{n}$; then $\mathcal{T}_{Q_{n}}$ is a monoid under composition.

For $k \geqslant 2$, a transformation (permutation) $t$ of a set $P=\left\{q_{0}, q_{1}, \ldots, q_{k-1}\right\} \subseteq Q$ is a $k$-cycle if $q_{0} t=q_{1}, q_{1} t=q_{2}, \ldots, q_{k-2} t=q_{k-1}, q_{k-1} t=q_{0}$. This $k$-cycle is denoted by $\left(q_{0}, q_{1}, \ldots, q_{k-1}\right)$. A 2-cycle $\left(q_{0}, q_{1}\right)$ is called a transposition. A transformation that changes only one state $p$ to a state $q \neq p$ is denoted by $(p \rightarrow q)$. A transformation mapping a subset $P$ of $Q$ to a single state and acting as the identity on $Q \backslash P$ is denoted by $(P \rightarrow q)$. We also denote by $\left[q_{0}, \ldots, q_{n-1}\right]$ the transformation that maps $p \in\{0, \ldots, n-1\}$ to $q_{p}$.

We now define dialects of languages and DFAs following [10]. Let $\Sigma=\left\{a_{1}, \ldots, a_{k}\right\}$ be an alphabet; we assume that its elements are ordered as shown. Let $\pi$ be a partial permutation of $\Sigma$, that is, a partial function $\pi: \Sigma \rightarrow \Gamma$ where $\Gamma \subseteq \Sigma$, for which there exists $\Delta \subseteq \Sigma$ such that $\pi$ is bijective when restricted to $\Delta$ and undefined on $\Sigma \backslash \Delta$. We denote undefined values of $\pi$ by the symbol "-_".

If $L$ is a language over $\Sigma$, we denote it by $L\left(a_{1}, \ldots, a_{k}\right)$ to stress its dependence on $\Sigma$. If $\pi$ is a partial permutation, let $s_{\pi}$ be the language substitution defined as follows: for $a \in \Sigma, a \mapsto\{\pi(a)\}$ when $\pi(a)$ is defined, and $a \mapsto \emptyset$ when $\pi(a)$ is not 
defined. For example, if $\Sigma=\{a, b, c\}, L(a, b, c)=\{a, b, c\}^{*}\{a b, a c c\}$, and $\pi(a)=c$, $\pi(b)=-$, and $\pi(c)=b$, then $s_{\pi}(L)=\{b, c\}^{*}\{c b b\}$. In other words, the letter $c$ plays the role of $a$, and $b$ plays the role of $c$. A permutational dialect of $L\left(a_{1}, \ldots, a_{k}\right)$ is a language of the form $s_{\pi}\left(L\left(a_{1}, \ldots, a_{k}\right)\right)$, where $\pi$ is a partial permutation of $\Sigma$; this dialect is denoted by $L\left(\pi\left(a_{1}\right), \ldots, \pi\left(a_{k}\right)\right)$. If the order on $\Sigma$ is understood, we use $L(\Sigma)$ for $L\left(a_{1}, \ldots, a_{k}\right)$ and $L(\pi(\Sigma))$ for $L\left(\pi\left(a_{1}\right), \ldots, \pi\left(a_{k}\right)\right)$. Undefined values appearing at the end of the alphabet are omitted. For example, if $\Sigma=\{a, b, c, d\}$ then we write $L(a, b)$ instead of $L(a, b,-,-)$.

Let $\Sigma=\left\{a_{1}, \ldots, a_{k}\right\}$, and let $\mathcal{D}=\left(Q, \Sigma, \delta, q_{1}, F\right)$ be a DFA; we denote it by $\mathcal{D}\left(a_{1}, \ldots, a_{k}\right)$ to stress its dependence on $\Sigma$. If $\pi$ is a partial permutation, then the permutational dialect

$$
\mathcal{D}\left(\pi\left(a_{1}\right), \ldots, \pi\left(a_{k}\right)\right)
$$

of $\mathcal{D}\left(a_{1}, \ldots, a_{k}\right)$ is obtained by changing the alphabet of $\mathcal{D}$ from $\Sigma$ to $\pi(\Sigma)$, and modifying $\delta$ so that in the modified DFA $\pi\left(a_{i}\right)$ induces the transformation induced by $a_{i}$ in the original DFA; thus $\pi\left(a_{i}\right)$ plays the role of $a_{i}$. One verifies that if the language $L\left(a_{1}, \ldots, a_{k}\right)$ is accepted by DFA $\mathcal{D}\left(a_{1}, \ldots, a_{k}\right)$, then $L\left(\pi\left(a_{1}\right), \ldots, \pi\left(a_{k}\right)\right)$ is accepted by $\mathcal{D}\left(\pi\left(a_{1}\right), \ldots, \pi\left(a_{k}\right)\right)$.

In the sequel we refer to permutational dialects simply as dialects.

\section{Contributions}

1. We prove that a most complex stream of suffix-free languages does not exist. This is in contrast with the existence of streams of most complex regular languages, right, left, and two-sided ideals, prefix-free and proper prefix-convex languages.

2. We exhibit a single ternary witness that meets the bounds for star, product, and Boolean operations.

3. We exhibit a single quinary witness that meets the bounds for Boolean operations, reversal, number of atoms, syntactic complexity, and quotient complexities of atoms.

4. We show that when $m, n \geqslant 6$ and $m-2$ and $n-2$ are relatively prime, there are binary witnesses that meet the bound $(m-1) 2^{n-2}+1$ for product.

5. We prove that any witness DFA for star and any second witness DFA for product must have transition semigroups that are subsemigroups of the suffix-free semigroup of transformations $\mathbf{T}^{\leqslant 5}(n)$ which has maximal cardinality for $2 \leqslant n \leqslant 5$; that the witness DFAs for reversal, syntactic complexity and quotient complexities of atoms must have transition semigroups that are subsemigroups of the suffix-free semigroup of transformations $\mathbf{T}^{\geqslant 6}(n)$ which has maximal cardinality for $n=2,3$ and $n \geqslant 6$; and that the witness DFAs for Boolean operations can have transition semigroups that are subsemigroups of $\mathbf{T}^{\leqslant 5} \cap \mathbf{T}^{\geqslant 6}$. 


\section{Suffix-Free Transformations}

In this section we discuss some properties of suffix-free languages with emphasis on their syntactic semigroups as represented by the transition semigroups of their quotient DFAs. We assume that our basic set is always $Q_{n}=\{0, \ldots, n-1\}$.

\subsection{Suffix-Free Languages}

Let $\mathcal{D}_{n}=\left(Q_{n}, \Sigma, \delta, 0, F\right)$ be the quotient DFA of a suffix-free language $L$, and let $T_{n}$ be its transition semigroup. For any transformation $t$ of $Q_{n}$, the sequence $\left(0,0 t, 0 t^{2}, \ldots\right)$ is called the 0 -path of $t$. Since $Q_{n}$ is finite, there exist $i, j$ such that $0,0 t, \ldots, 0 t^{i}, 0 t^{i+1}, \ldots, 0 t^{j-1}$ are distinct but $0 t^{j}=0 t^{i}$. The integer $j-i$ is the period of $t$ and if $j-i=1, t$ is initially aperiodic. The following properties of suffix-free languages are known [12, 26]:

Lemma 1. If $L$ is a regular suffix-free language, then

1. There exists $w \in \Sigma^{*}$ such that $w^{-1} L=\emptyset$; hence $\mathcal{D}_{n}$ has an empty state, which is state $n-1$ by convention.

2. For $w, x \in \Sigma^{+}$, if $w^{-1} L \neq \emptyset$, then $w^{-1} L \neq(x w)^{-1} L$.

3. If $L \neq \emptyset$ and $w^{-1} L=L$, then $w=\varepsilon$.

4. For any $t \in T_{n}$, the 0 -path of $t$ in $\mathcal{D}_{n}$ is aperiodic and ends in $n-1$.

Property 3 is known as the non-returning property [26] and also as unique reachability [11].

An (unordered) pair $\{p, q\}$ of distinct states in $Q_{n} \backslash\{0, n-1\}$ is colliding (or $p$ collides with $q$ ) in $T_{n}$ if there is a transformation $t \in T_{n}$ such that $0 t=p$ and $r t=q$ for some $r \in Q_{n} \backslash\{0, n-1\}$. A pair of states is focused by a transformation $u$ of $Q_{n}$ if $u$ maps both states of the pair to a single state $r \notin\{0, n-1\}$. We then say that $\{p, q\}$ is focused to state $r$. If $L$ is a suffix-free language, then from Lemma 1 (2) it follows that if $\{p, q\}$ is colliding in $T_{n}$, there is no transformation $t^{\prime} \in T_{n}$ that focuses $\{p, q\}$. So colliding states can be mapped to a single state by a transformation in $T_{n}$ only if that state is the empty state $n-1$.

Following [12], for $n \geqslant 2$, we let

$$
\begin{aligned}
\mathbf{B}(n)=\left\{t \in \mathcal{T}_{Q} \mid\right. & 0 \notin \operatorname{rng}(t),(n-1) t=n-1, \text { and for all } j \geqslant 1, \\
& \left.0 t^{j}=n-1 \text { or } 0 t^{j} \neq q t^{j}, \forall q \text { such that } 0<q<n-1\right\} .
\end{aligned}
$$

Example 1. We have $\mathbf{B}(2)=\{[1,1]\}$ and $\mathbf{B}(3)=\{[1,2,2],[2,1,2],[2,2,2]\}$. For $n=4$, there are 17 transformations satisfying $0 t \neq q t$. However, if $t=[1,2,2,3]$ or $t=[2,1,1,3]$, then $0 t^{2}=1 t^{2}$, which violates the third condition for $\mathbf{B}$; hence $\mathbf{B}(4)$ has 15 elements. The cardinality of $\mathbf{B}(5)$ is 115 . 
Proposition 1 ([12]). If $L$ is a regular language with $\mathcal{D}_{n}=\left(Q_{n}, \Sigma, \delta, 0, F\right)$ as its minimal DFA and syntactic semigroup $T_{L}$, then the following hold:

1. If $L$ is suffix-free, then $T_{L}$ is a subset of $\mathbf{B}(n)$.

2. If $L$ has the empty quotient, only one final quotient, and $T_{L} \subseteq \mathbf{B}(n)$, then $L$ is suffix-free.

Since the transition semigroup of a minimal DFA of a suffix-free language must be a subsemigroup of $\mathbf{B}(n)$, the cardinality of $\mathbf{B}(n)$ is an upper bound on the syntactic complexity of suffix-free regular languages with quotient complexity $n$. This upper bound, however, cannot be reached since $\mathbf{B}$ is not a semigroup for $n \geqslant 4$ : We have $s=[1,2, n-1, \ldots, n-1]$ and $t=[n-1,2,2, \ldots, 2, n-1]$ in $\mathbf{B}(n)$, but $s t=[2,2, n-1, \ldots, n-1]$ is not in $\mathbf{B}(n)$.

\subsection{Semigroups $\mathbf{T}^{\leqslant 5}(n)$ with Maximal Cardinality when $2 \leqslant n \leqslant 5$}

For $n \geqslant 2$, let

$$
\begin{aligned}
\mathbf{T}^{\leqslant 5}(n)=\{t \in \mathbf{B}(n) \mid & \text { for all } p, q \in Q_{n} \text { where } p \neq q \\
& \text { either } p t=q t=n-1 \text { or } p t \neq q t\} .
\end{aligned}
$$

Proposition 2. For $n \geqslant 4$, the semigroup $\mathbf{T}^{\leqslant 5}(n)$ is generated by the following set $\mathbf{H}^{\leqslant 5}(n)$ of transformations of $Q$ :

- $a:(0 \rightarrow n-1)(1, \ldots, n-2)$,

- $b:(0 \rightarrow n-1)(1,2)$,

- for $1 \leqslant p \leqslant n-2, c_{p}:(p \rightarrow n-1)(0 \rightarrow p)$.

For $n=4$, $a$ and $b$ coincide, and so $\mathbf{H}^{\leqslant 5}(4)=\left\{a, c_{1}, c_{2}\right\}$. Also, $\mathbf{H}^{\leqslant 5}(3)=\left\{a, c_{1}\right\}=$ $\{[2,1,2],[1,2,2]\}$ and $\mathbf{H}^{\leqslant 5}(2)=\left\{c_{1}\right\}=\{[1,1]\}$.

Proof. It was proved in [12] that for $n \geqslant 4$, the semigroup $\mathbf{T}^{\leqslant 5}(n)$ is generated by the following set $\mathbf{G}^{\leqslant 5}(n)$ of $n$ transformations of $Q_{n}$ : $a$ and $b$ as above, and $c_{p}^{\prime}$ for $1 \leqslant p \leqslant n-2$, defined by $q c_{p}^{\prime}=q+1$ for $q=0, \ldots, p-1, p c_{p}^{\prime}=n-1$, and $q c_{p}^{\prime}=q$ for $q=p+1 \ldots, n-1$. Since $\mathbf{H}^{\leqslant 5}(n) \subseteq \mathbf{T}^{\leqslant 5}$, the semigroup generated by $\mathbf{H}^{\leqslant 5}(n)$ is a subsemigroup of $\mathbf{T}^{\leqslant 5}(n)$. So it is sufficient to show that every transformation in $\mathbf{G}^{\leqslant 5}(n)$ can be generated by $\mathbf{H}^{\leqslant 5}(n)$. Transformation $c_{p}^{\prime}$ changes $\{0,1, \ldots, p-1\}$ to $\{1,2, \ldots, p\}, p$ is mapped to $n-1$, and $\{p+1, p+2, \ldots, n-2)$ is mapped to itself. The image of $Q_{n} \backslash\{p, n-1\}$ is thus $\{1,2, \ldots, p, p+1, \ldots n-2\}$. The image of $Q_{n} \backslash\{p, n-1\}$ under $c_{p}$ is $\{p, 1,2, \ldots, p-1, p+1, \ldots, n-2\}$. However, since transformations $a$ 
and $b$ restricted to $Q_{n} \backslash\{0, n-1\}$ generate all permutations of $Q_{n} \backslash\{0, n-1\}$, $\{p, 1,2, \ldots, p-1, p+1, \ldots, n-2\}$ can be transformed to $\{1,2, \ldots, p, p+1, \ldots n-2\}$. Hence the claim holds.

From now on we use the transformations of Proposition 2 for $\mathbf{T}^{\leqslant 5}(n)$. A DFA using these transformations is illustrated in Figure 2.

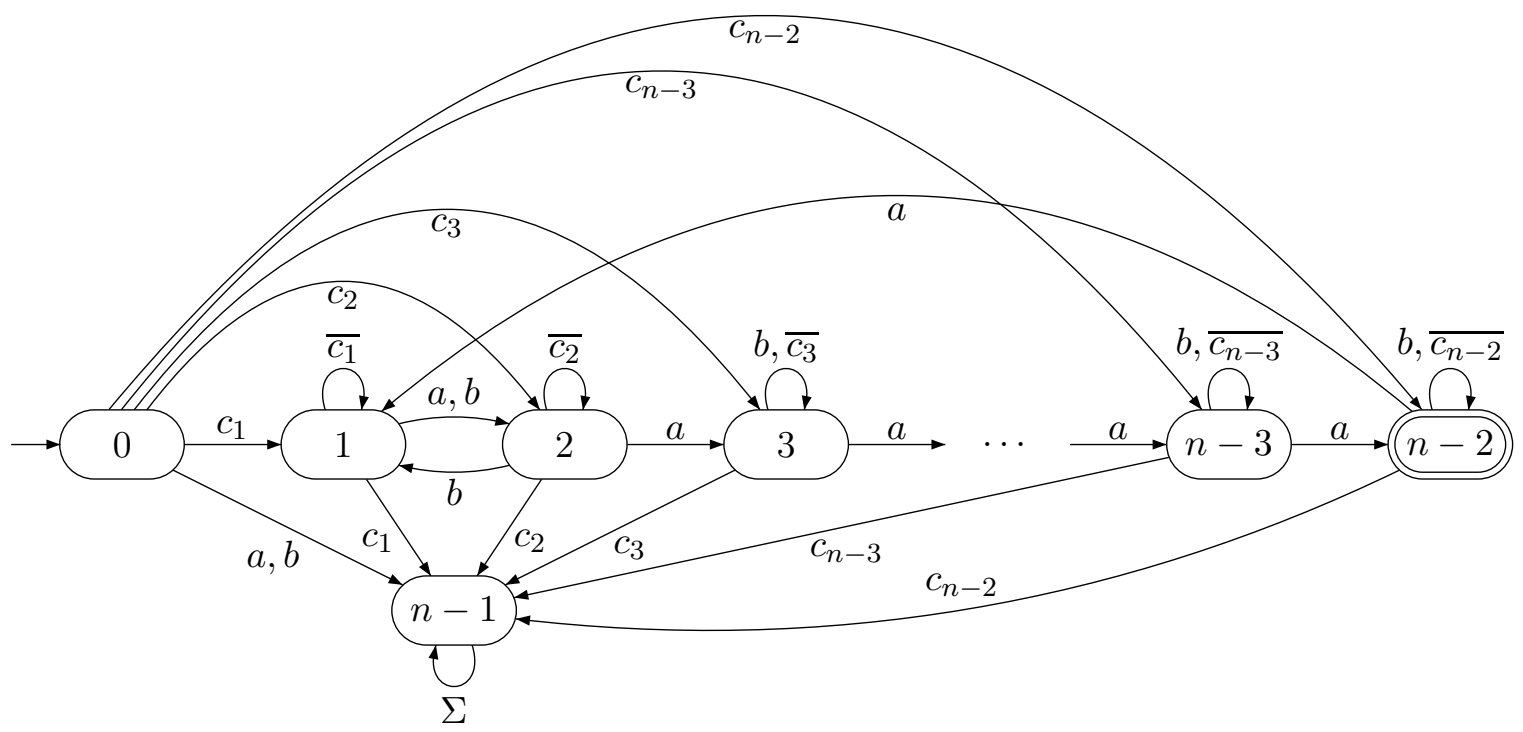

Figure 2: DFA with $\mathbf{T}^{\leqslant 5}(n)$ as its transformation semigroup; $\overline{c_{p}}=\left\{c_{1}, \ldots, c_{n-2}\right\} \backslash\left\{c_{p}\right\}$.

Proposition 3. For $n \geqslant 2, \mathbf{T}^{\leqslant 5}(n)$ is the unique maximal semigroup of a suffix-free language in which all possible pairs of states are colliding.

Proof. For each pair $p, q \in Q \backslash\{0, n-1\}, p \neq q$, there is a transformation $c_{p} \in \mathbf{T}^{\leqslant 5}(n)$ with $0 c_{p}=p$ and $q c_{p}=q$. Thus all pairs are colliding. If all pairs are colliding, then for each $p, q \in Q \backslash\{n-1\}$, there is no transformation $t$ with $p t=q t \neq n-1$, for this would violate suffix-freeness. By definition, $\mathbf{T}^{\leqslant 5}(n)$ has all other transformations that are possible for a suffix-free language, and hence is unique.

Proposition 4. For $n \geqslant 5$, the number $n$ of generators of $\mathbf{T}^{\leqslant 5}(n)$ cannot be reduced.

Proof. If a generator $t$ maps 0 to $p \in\{1, \ldots, n-2\}$, then it must also map a state $q \in\{1, \ldots, n-2\}$ to $n-1$, since the 0 -path of $t$ is aperiodic and ends in $n-1$. Thus $t$ cannot generate a permutation of $\{1, \ldots, n-2\}$. Since $\mathbf{T}^{\leqslant 5}(n)$ has all transformations 
that map 0 to $n-1$, permute $\{1, \ldots, n-2\}$ and fix $n-1$, we need two generators, say $a$ and $b$, with $0 a=0 b=n-1$ to induce all the permutations.

For $p \in\{1, \ldots, n-2\}$, consider the transformation $c_{p}$ of Proposition 2. Note that every transformation that maps 0 to a state in $\{1, \ldots, n-2\}$ must also map a state in $\{1, \ldots, n-2\}$ to $n-1$. Since $c_{p}$ maps $\{0,1, \ldots, n-2\} \backslash\{p\}$ onto $\{1, \ldots, n-2\}$, it must be generated by $c_{p}^{\prime} u$, where $c_{p}^{\prime}$ is a transformation mapping 0 to some state $q \in\{1, \ldots, n-2\}$ and $p$ to $n-1$, and where $u$ permutes $\{1, \ldots, n-2\}$. Since each $c_{p}$ requires a different $c_{p}^{\prime}$, one for each $p \in\{1, \ldots, n-2\}$, we need at least $n-2$ generators $c_{p}^{\prime}$.

Example 2. $\mathbf{T}^{\leqslant 5}(4)$ has 13 elements. All transitions of $\mathbf{B}(4)$ are present except $[3,1,1,3]$ and $[3,2,2,3]$. The semigroup $\mathbf{T}^{\leqslant 5}(5)$ has 73 elements.

Semigroups $\mathbf{T}^{\leqslant 5}(n)$ are suffix-free semigroups that have maximal cardinality when $2 \leqslant n \leqslant 5[12]$.

2.3. Semigroups $\mathbf{T}^{\geqslant 6}(n)$ with Maximal Cardinality when $n=2,3$ and $n \geqslant 6$

For $n \geqslant 2$, let

$$
\begin{aligned}
& \mathbf{T}^{\geqslant 6}(n)=\{t \in \mathbf{B}(n) \quad \mid \quad 0 t=n-1, \text { or } \\
& q t=n-1 \text { for all } q \text { such that } 1 \leqslant q \leqslant n-2\} .
\end{aligned}
$$

Proposition 5 ([20]). For $n \geqslant 4, \mathbf{T}^{\geqslant 6}(n)$ is a semigroup contained in $\mathbf{B}(n)$, its cardinality is $(n-1)^{n-2}+(n-2)$, and it is generated by the set $\mathbf{G}^{\geqslant 6}(n)$ of the following transformations:

- $a:(0 \rightarrow n-1)(1, \ldots, n-2)$;

- $b:(0 \rightarrow n-1)(1,2)$;

- $c:(0 \rightarrow n-1)(n-2 \rightarrow 1)$;

- $d:(\{0,1\} \rightarrow n-1)$;

- $e:(Q \backslash\{0\} \rightarrow n-1)(0 \rightarrow 1)$.

For $n=4$, $a$ and $b$ coincide, and so $\mathbf{G}^{\geqslant 6}(4)=\{a, c, d, e\}$. Also $\mathbf{G}^{\geqslant 6}(3)=\{a, e\}=$ $\{[2,1,2],[1,2,2]\}$ and $\mathbf{G}^{\geqslant 6}(2)=\{e\}=\{[1,1]\}$.

Example 3. $\mathbf{T}^{\geqslant 6}(4)$ has 11 elements. All transitions of $\mathbf{B}(4)$ are present except $[1,2,3,3],[1,3,2,3],[2,1,3,3]$ and $[2,3,1,3]$. Semigroup $\mathbf{T}^{\geqslant 6}(5)$ has size 67.

A DFA using the transformations of Proposition 5 is shown in Figure 3.

Semigroups $\mathbf{T}^{\geqslant 6}(n)$ are suffix-free semigroups that have maximal cardinality when $n \geqslant 6[20]$. 


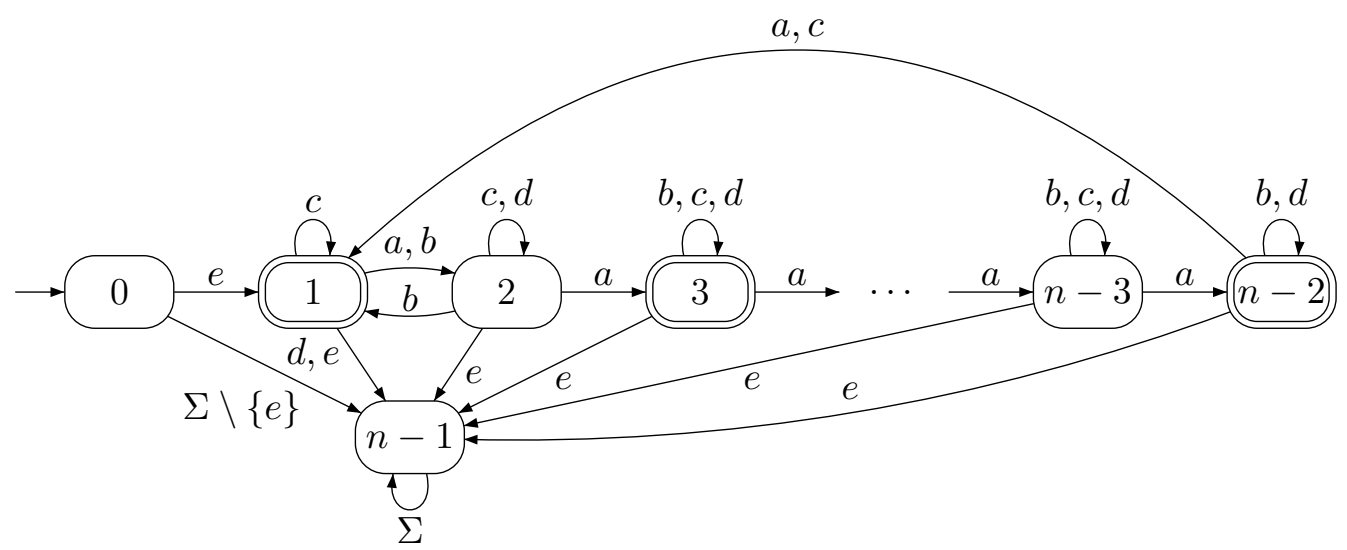

Figure 3: DFA with $\mathbf{T}^{\geqslant 6}(n)$ as its transformation semigroup for the case when $n$ is odd.

\section{Witnesses with Transition Semigroups in $T^{\leqslant 5}(n)$}

In this section we consider DFA witnesses whose transition semigroups are subsemigroups of $\mathbf{T}^{\leqslant 5}(n)$. We show that there is one witness that satisfies the bounds for star, product and Boolean operations.

Definition 1. For $n \geqslant 6$, we define the DFA $\mathcal{D}_{n}=\left(Q_{n}, \Sigma, \delta, 0,\{1\}\right)$, where $Q_{n}=$ $\{0, \ldots, n-1\}, \Sigma=\{a, b, c\}$, and $\delta$ is defined by the transformations

- $a:(0 \rightarrow n-1)(1,2,3)(4, \ldots, n-2)$,

- $b:(2 \rightarrow n-1)(1 \rightarrow 2)(0 \rightarrow 1)(3,4)$,

- $c:(0 \rightarrow n-1)(1, \ldots, n-2)$.

Theorem 1 (Star, Product, Boolean Operations). Let $\mathcal{D}_{n}(a, b, c)$ be the DFA of Definition 1, and let the language it accepts be $L_{n}(a, b, c)$. For $n \geqslant 6, L_{n}$ and its permutational dialects meet the bounds for star, product and Boolean operations as follows:

1. $L_{n}^{*}(a, b,-)$ meets the bound $2^{n-2}+1$. [Cmorik and Jirásková [23]]

2. $L_{m}(a, b, c) \cdot L_{n}(b, c, a)$ meets the bound $(m-1) 2^{n-2}+1$.

3. $L_{m}(a, b,-)$ and $L_{n}(-, b, a)$ meet the bounds $m n-(m+n-2)$ for union and symmetric difference, $m n-2(m+n-3)$ for intersection, and $m n-(m+2 n-4)$ for difference.

The claim about the star operation was proved in [23]. We add a result about the transition semigroup of the star witness and prove the remaining two claims in this section. 


\subsection{Star}

In 2009 Han and Salomaa [26] showed that the language of a DFA over a fourletter alphabet meets the bound $2^{n-2}+1$ for the star operation for $n \geqslant 4$. The transition semigroup of this DFA is a subsemigroup of $\mathbf{T}^{\leqslant 5}(n)$. In 2012 Cmorik and Jirásková [23] showed that for $n \geqslant 6$ a binary alphabet $\{a, b\}$ suffices. The transition semigroup of this DFA is again a subsemigroup of $\mathbf{T}^{\leqslant 5}(n)$. We prove that these are special cases of the following general result:

Theorem 2. For $n \geqslant 4$, the transition semigroup of a minimal $D F A \mathcal{D}\left(Q_{n}, \Sigma, \delta, 0, F\right)$ of a suffix-free language $L$ that meets the bound $2^{n-2}+1$ for the star operation is a subsemigroup of $\mathbf{T}^{\leqslant 5}(n)$ and is not a subsemigroup of $\mathbf{T}^{\geqslant 6}(n)$.

Proof. To show that the transition semigroup of $\mathcal{D}$ is a subsemigroup of $\mathbf{T}^{\leqslant 5}(n)$, by Proposition 3 it suffices to show that every pair of states is colliding.

We construct an NFA $\mathcal{N}$ for $L_{n}^{*}$ by making 0 a final state in $\mathcal{D}$ - this is possible since 0 is uniquely reachable - and adding an empty-word transition from every final state to 0 . We then determinize $\mathcal{N}$ using the subset construction to get a DFA $\mathcal{D}^{*}$ for $L_{n}^{*}$. The states of $\mathcal{D}^{*}$ are sets of states of $\mathcal{D}$.

Consider a subset $S \subseteq Q_{n}$. We can assume that $n-1 \in S$, since $S$ and $S \cup\{n-1\}$ cannot be distinguished. If $S \neq\{0, n-1\}$ and $S \neq\{n-1\}$, then $S$ can be reached only if $0 \in S$ and $S \cap F \neq \emptyset$, or $0 \notin S$ and $S \cap F=\emptyset$, because by the construction for star in $\mathcal{N}$ there is an $\varepsilon$-transition from every final state to the initial state 0 and no transformation fixes 0 . Thus, to meet the bound $2^{n-2}+1$, for each possible subset of $\{1, \ldots, n-2\}$ there must be a reachable subset $S$ containing that subset.

Suppose that $p, q \in\{1, \ldots, n-2\}$ are not colliding, that is, there is no transformation $t$ with $0 t=p$ and $q \in \operatorname{rng}(t)$. Consider $S$ that contains both $p$ and $q$. Since the $\varepsilon$-transitions from final states to initial state 0 are the only sources of nondeterminism, $S$ must be reached from a subset $S^{\prime}$ containing 0 and $q^{\prime} \in\{1, \ldots, n-2\}$ by a transformation $t$ with $0 t=p$ and $q^{\prime} t=q\left(\right.$ or $0 t=q$ and $\left.q^{\prime} t=p\right)$, which contradicts that $p, q$ are not colliding.

Since transformations causing colliding pairs are not in $\mathbf{T}^{\geqslant 6}(n)$, the transition semigroup of $\mathcal{D}$ cannot be a subsemigroup of $\mathbf{T}^{\geqslant 6}(n)$.

\subsection{Product}

To avoid confusing the states of the two DFAs in a product, we label the states

of the first DFA differently. Let $\mathcal{D}_{m}^{\prime}=\mathcal{D}_{m}^{\prime}(a, b, c)=\left(Q_{m}^{\prime}, \Sigma, \delta^{\prime}, 0^{\prime},\left\{1^{\prime}\right\}\right)$, where $Q_{m}^{\prime}=\left\{0^{\prime}, \ldots,(m-1)^{\prime}\right\}$, and $\delta^{\prime}\left(q^{\prime}, x\right)=p^{\prime}$ if $\delta(q, x)=p$, and let $\mathcal{D}_{n}=\mathcal{D}_{n}(b, c, a)$. We use the standard construction of the $\varepsilon$-NFA $\mathcal{N}$ for the product: the final state 


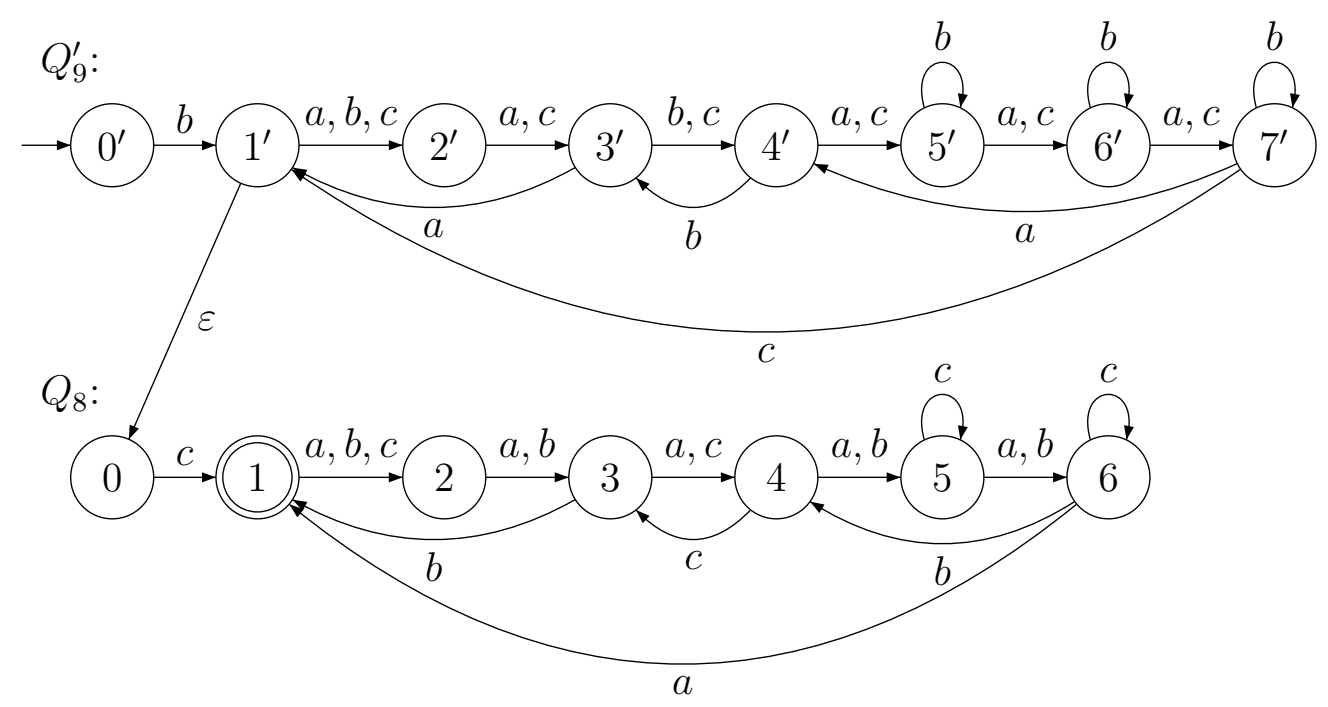

Figure 4: The NFA $\mathcal{N}$ for product $L_{9}^{\prime}(a, b, c) \cdot L_{8}(b, c, a)$. The empty states $8^{\prime}$ and 7 and the transitions to them are omitted.

of $\mathcal{D}_{m}^{\prime}$ becomes non-final, and an $\varepsilon$-transition is added from that state to the initial state of $\mathcal{D}_{n}$. This is illustrated in Figure 4 for $m=9, n=8$.

We use the subset construction to determinize $\mathcal{N}$ to get a DFA $\mathcal{P}$ for the product. The reachable states of $\mathcal{P}$ are subsets of $Q_{m}^{\prime} \cup Q_{n}$ and have one of three forms: $\left\{0^{\prime}\right\}$, $\left\{1^{\prime}, 0\right\} \cup S$ or $\left\{p^{\prime}\right\} \cup S$, where $p^{\prime}=2^{\prime}, \ldots,(m-1)^{\prime}$ and $S \subseteq\{1, \ldots, n-1\}$.

Note that for each $x \in \Sigma$ every state $q \in Q_{n} \backslash\{0, n-1\}$ has a unique predecessor state $p \in Q_{n} \backslash\{n-1\}$ such that $p x=q$. For $w \in \Sigma^{*}$, the $w$-predecessor of $S \subseteq Q_{n} \backslash\{0, n-1\}$ is denoted by $S w^{-1}$.

Lemma 2. For each $n \geqslant 6$ and each $q \in Q_{n}$ there exists a word $w_{q} \in c\{a, b\}^{*}$ such that $1^{\prime} w_{q}=3^{\prime}, 0 w_{q}=q$, and each state of $Q_{n} \backslash\{0, q, n-1\}$ has a unique $w_{q}$-predecessor in $Q_{n} \backslash\{0, n-1\}$. In fact, the following $w_{q}$ satisfy these requirements:

$$
w_{q}= \begin{cases}c a b^{2}, & \text { if } q=1 ; \\ c a, & \text { if } q=2 ; \\ c a b^{4}, & \text { if } q=3 ; \\ c a b^{2} a^{3} b^{q-4}, & \text { if } q \geqslant 4 \text { and } q \text { is even } \\ c a^{4} b^{q-5}, & \text { if } q \geqslant 5 \text { and } q \text { is odd. }\end{cases}
$$


Proof. It is easily verified that in each case $1^{\prime} w_{q}=3^{\prime}$ and $0 w_{q}=q$. Note that $a$ and $b$ induce permutations on $Q_{n} \backslash\{0, n-1\}$ and $c$ is a one-to-one mapping from $Q_{n} \backslash\{0, n-1\}$ to $Q_{n} \backslash\{0,1\}$. Thus every state in $Q_{n} \backslash\{0, n-1\}$ that is mapped by $w_{q}$ to $Q_{n} \backslash\{0, n-1\}$ has a $w_{q}$-predecessor in $Q_{n} \backslash\{0, n-1\}$, and state $q$ has 0 as its $w_{q}$-predecessor.

Theorem 3 (Product: Ternary Case). For $m, n \geqslant 6$, the product $L_{m}^{\prime}(a, b, c)$. $L_{n}(b, c, a)$ meets the bound $(m-1) 2^{n-2}+1$.

Proof. Let $P$ consist of the following states $\left\{0^{\prime}\right\}, 2^{n-2}$ sets of the form $\left\{1^{\prime}, 0, n-1\right\} \cup S$ and $(m-2) 2^{n-2}$ sets of the form $\left\{p^{\prime}, n-1\right\} \cup S$, where $S \subseteq\{1, \ldots, n-2\}$, and $p^{\prime}=2^{\prime}, \ldots,(m-1)^{\prime}-$ a total of $(m-1) 2^{n-2}+1$ sets. We shall prove that all these states of $\mathcal{P}$ are reachable and pairwise distinguishable. This together with the known upper bound will prove that the witnesses indeed meet the bound for product.

Consider the distinguishability of two states in $P$. If we apply any word ending in $c$ to any subset of $Q_{n} \backslash\{0\}$, the resulting set does not contain the final state 1 . Suppose that one of the states in our pair is $\left\{0^{\prime}\right\}$; this is the only state accepting $b c$. Next, if one of the states has the form $\left\{1^{\prime}, 0, n-1\right\} \cup S$ and the other is $\left\{p^{\prime}, n-1\right\} \cup R$, then the former state accepts $c$, whereas the latter does not. If $p<q$, then $\left\{p^{\prime}, n-1\right\} \cup R$ is distinguished from $\left\{q^{\prime}, n-1\right\} \cup S$ by $c^{m-q}$. This leaves the case where the state in $Q_{m}^{\prime} \backslash\left\{0^{\prime}\right\}$ is the same in both sets in our pair. If the sets in $Q_{n}$ are $R$ and $S, R \neq S$, and $q \in R \oplus S$, then $a^{m-1-q}$ distinguishes these states.

Now we turn to reachability. Since state $0^{\prime}$ is initial in $\mathcal{N},\left\{0^{\prime}\right\}$ is reachable. First we show that the sets in $P$ are reachable if $S=\emptyset$. The set $\left\{1^{\prime}, 0, n-1\right\}$ is reached by $b a^{3},\left\{2^{\prime}, n-1\right\}$ by $b a,\left\{p^{\prime}, n-1\right\}$ by bac $c^{p-2}$ for $p=3, \ldots,(m-2)$, and $\left\{(m-1)^{\prime}, n-1\right\}$ by $b^{3}$.

Now suppose all sets of the form $\left\{1^{\prime}, 0, n-1\right\} \cup S$ and $\left\{p^{\prime}, n-1\right\} \cup S, p=$ $2, \ldots, m-1$, with $S \subseteq\{1, \ldots, n-2\}$ and $|S|=k$, are reachable. We show that if $k<n-2$, then every set with $S$ of size $k+1$ can be reached. In each case we assume that $q \notin S$.

1. Sets with $3^{\prime}$. We add $q$ to $S$ by applying $w_{q}$ to $\left(\left\{1^{\prime}, 0, n-1\right\} \cup S w_{q}^{-1}\right)$. By Lemma 2 , every state except $q$ in $Q_{n} \backslash\{0, n-1\}$ has a unique $w_{q}$-predecessor in $Q_{n} \backslash\{0, n-1\}$. Hence, assuming we have $\left\{3^{\prime}, n-1\right\} \cup S w_{q}^{-1}$ we can add $q$ since

$$
\left(\left\{1^{\prime}, 0, n-1\right\} \cup S w_{q}^{-1}\right) w_{q}=\left\{3^{\prime}, n-1\right\} \cup S \cup\{q\} .
$$

2. Sets with $4^{\prime}$ :

(a) $\left(\left\{3^{\prime}, n-1\right\} \cup S b^{-1} \cup\{3\}\right) b=\left\{4^{\prime}, n-1\right\} \cup S \cup\{1\}$,

(b) $\left(\left\{3^{\prime}, n-1\right\} \cup S b^{-1} \cup\{1\}\right) b=\left\{4^{\prime}, n-1\right\} \cup S \cup\{2\}$, 
(c) $\left(\left\{3^{\prime}, n-1\right\} \cup S b^{-1} \cup\{2\}\right) b=\left\{4^{\prime}, n-1\right\} \cup S \cup\{3\}$,

(d) $\left(\left\{3^{\prime}, n-1\right\} \cup S b^{-1} \cup\{m-2\}\right) b=\left\{4^{\prime}, n-1\right\} \cup S \cup\{4\}$,

(e) $\left(\left\{3^{\prime}, n-1\right\} \cup S b^{-1} \cup\{q\}\right) b=\left\{4^{\prime}, n-1\right\} \cup S \cup\{q+1\}$, for $q=4, \ldots, n-2$.

3. Sets with $p^{\prime}, p=5, \ldots, m-2$ :

$$
\begin{gathered}
\left(\left\{(p-1)^{\prime}, n-1\right\} \cup S\left(b^{2} a b\right)^{-1} \cup\{3\}\right) b^{2} a b=\left\{p^{\prime}, n-1\right\} \cup S \cup\{1\}, \\
\left(\left\{(p-1)^{\prime}, n-1\right\} \cup S a^{-1} \cup\{q-1\}\right) a=\left\{p^{\prime}, n-1\right\} \cup S \cup\{q\} \text { for } q=2, \ldots, n-2 .
\end{gathered}
$$

4. Sets with $(m-1)^{\prime}$ : If $1 \notin S$, then $S$ has a $c$-predecessor in $Q_{n} \backslash\{0, n-1\}$ and $\left(\left\{1^{\prime}, 0, n-1\right\} \cup S c^{-1}\right) c=\left\{2^{\prime}, n-1\right\} \cup S \cup\{1\}$. Thus we have

$$
\begin{gathered}
\left(\left\{2^{\prime}, n-1\right\} \cup S(b a b)^{-1} \cup\{1\}\right) b a b=\left\{(m-1)^{\prime}, n-1\right\} \cup S \cup\{1\}, \\
\left(\left\{2^{\prime}, n-1\right\} \cup S b^{-1} \cup\{1\}\right) b=\left\{(m-1)^{\prime}, n-1\right\} \cup S \cup\{2\}, \\
\left(\left\{(m-1)^{\prime}, n-1\right\} \cup S a^{-1} \cup\{q-1\}\right) a=\left\{(m-1)^{\prime}, n-1\right\} \cup S \cup\{q\},
\end{gathered}
$$

for $q=3, \ldots, n-2$.

5. Sets with $1^{\prime}$ :

$$
\begin{gathered}
\left(\left\{3^{\prime}, n-1\right\} \cup S a^{-1} \cup\{n-2\}\right) a=\left\{1^{\prime}, 0, n-1\right\} \cup S \cup\{1\}, \\
\left(\left\{3^{\prime}, n-1\right\} \cup S a^{-1} \cup\{1\}\right) a=\left\{1^{\prime}, 0, n-1\right\} \cup S \cup\{2\}, \\
\left(\left\{2^{\prime}, n-1\right\} \cup S\left(a^{2}\right)^{-1} \cup\{1\}\right) a^{2}=\left\{1^{\prime}, 0, n-1\right\} \cup S \cup\{3\}, \\
\left(\left\{1^{\prime}, 0, n-1\right\} \cup S\left(a^{3}\right)^{-1} \cup\{q-3\}\right) a^{3}=\left\{1^{\prime}, 0, n-1\right\} \cup S \cup\{q\},
\end{gathered}
$$

for $q=4, \ldots, n-2$.

6. Sets with $2^{\prime}$ :

$$
\begin{gathered}
\left(\left\{1^{\prime}, 0, n-1\right\} \cup S c^{-1}\right) c=\left\{2^{\prime}, n-1\right\} \cup S \cup\{1\}, \text { as in Case 4, } \\
\left(\left\{1^{\prime}, 0, n-1\right\} \cup S a^{-1} \cup\{q-1\}\right) a=\left\{2^{\prime}, n-1\right\} \cup S \cup\{q\},
\end{gathered}
$$

for $q=2, \ldots, n-2$.

Thus the induction step goes through and all required sets are reachable.

Cmorik and Jirásková [23, Theorem 5] also found binary witnesses that meet the bound $(m-1) 2^{n-2}$ in the case where $m-2$ and $n-2$ are relatively prime. It remained unknown whether the bound $(m-1) 2^{n-2}+1$ is reachable with a binary alphabet. We show in the Appendix that a slight modification of the first witness of [23] meets the upper bound exactly. 
Theorem 4. Suppose $m, n \geqslant 4$ and $L_{m}^{\prime} L_{n}$ meets the bound $2^{n-2}+1$. Then the transition semigroup $T_{n}$ of a minimal DFA $\mathcal{D}_{n}$ of $L_{n}$ is a subsemigroup of $\mathbf{T}^{\leqslant 5}(n)$ and is not a subsemigroup of $\mathbf{T}^{\geqslant 6}(n)$.

Proof. Let $\mathcal{D}_{m}^{\prime}=\left(Q_{m}^{\prime}, \Sigma, \delta^{\prime}, 0^{\prime}, F^{\prime}\right)$ with $Q_{m}^{\prime}=\left\{0^{\prime}, \ldots,(m-1)^{\prime}\right\}$, and let $\mathcal{D}_{n}=$ $\left(Q_{n}, \Sigma, \delta, 0, F\right)$ with $Q_{n}=\{0, \ldots, n-1\}$. We construct the NFA for $L_{m}^{\prime} L_{n}$ as usual.

From [26, Lemma 9] we know that the set of reachable and distinguishable subsets of $Q_{m}^{\prime} \cup Q_{n}$ can be represented by: $\left\{p^{\prime}\right\} \cup X$ for each $p \in\{1, \ldots, m-1\}$ and $X \subseteq\{1, \ldots, n-2\}$, or by $\left\{0^{\prime}\right\}$. A reachable subset $S$ contains 0 if and only if $S \cap F^{\prime} \neq \emptyset$. Also $S$ is not distinguishable from $S \cup\left\{(m-1)^{\prime}\right\}$ and $S \cup\{n-1\}$. To reach the bound $(m-1) 2^{n-2}+1$, all these subsets or their equivalents must be reachable.

Suppose that $p, q \in\{1, \ldots, n-2\}$ are not colliding in $Q_{n}$. Consider $S$ that contains both $p$ and $q$. Then $S$ is reached from some $S^{\prime}$ by a transformation $t$, where $S^{\prime}$ contains 0 and a state $r \in\{1, \ldots, n-2\}$ such that $0 t=p$ and $r t=q$ (or $0 t=q$ and $r t=p$ ). But since $p$ and $q$ are not colliding, there is no such transformation in $T_{n}$. Thus all pairs in $Q_{n} \backslash\{0, n-1\}$ must be colliding and $T_{n}$ is a subsemigroup of $\mathbf{T}^{\leqslant 5}$.

\subsection{Boolean Operations}

Let $\mathcal{D}=\mathcal{D}(a, b, c)$ be the DFA of Definition 1 , and let $L=L(a, b, c)$ be the language of this DFA. Consider the partial permutation $\pi^{\prime}(a)=a, \pi^{\prime}(b)=b$, and $\pi^{\prime}(c)=-$. The dialect associated with $\pi^{\prime}$ is $\mathcal{D}^{\prime}(a, b,-)$, which is $\mathcal{D}(a, b, c)$ restricted to the alphabet $\{a, b\}$. This is our first witness for Boolean operations, and we prime its states to distinguish them from those of the second witness defined below. This DFA is illustrated in Figure 5 for $m=9$.

Now take the partial permutation $\pi$, where $\pi(a)=-, \pi(b)=b$, and $\pi(c)=a$; here $a$ plays the role of $c$. Thus $\mathcal{D}(-, b, a)$ is the DFA in which $a:(0 \rightarrow n-1)(1, \ldots, n-2)$ and $b:(2 \rightarrow n-1)(1 \rightarrow 2)(0 \rightarrow 1)(3,4)$, as illustrated in Figure 5 for $n=8$. This DFA is our second witness for Boolean operations. The language of $\mathcal{D}(-, b, a)$ is the dialect $L(-, b, a)$ of $L$.

Theorem 5. For $m, n \geqslant 6, L_{m}^{\prime}(a, b,-)$ and $L_{n}(-, b, a)$ meet the bounds $m n-(m+$ $n-2)$ for union and symmetric difference, $m n-2(m+n-3)$ for intersection, and $m n-(m+2 n-4)$ for difference.

Proof. As usual, we construct the direct product $\mathcal{P}$ of $\mathcal{D}_{m}^{\prime}$ and $\mathcal{D}_{n}$. The initial state of $\mathcal{P}$ is $\left(0^{\prime}, 0\right)$ which reaches only $\left(1^{\prime}, 1\right)$ and can never be re-entered. Thus $0^{\prime}$ and 0 do not appear in any reachable state of $\mathcal{P}$ other than $\left(0^{\prime}, 0\right)$. We show that the remaining 


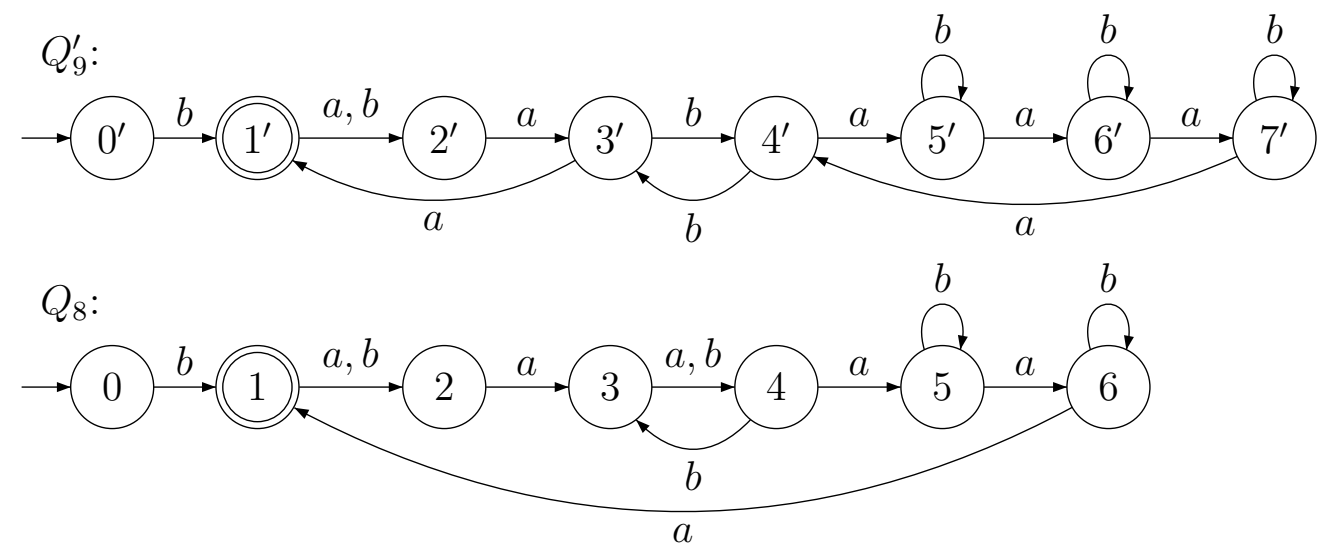

Figure 5: Witnesses $\mathcal{D}_{9}^{\prime}(a, b,-)$ and $\mathcal{D}_{8}(-, b, a)$ for Boolean operations. The empty states $8^{\prime}$ and 7 and the transitions to them are omitted.

$(m-1)(n-1)$ states are all reachable, for a total of $1+(m-1)(n-1)=m n-(m+n-2)$ states. Let $m$ define the row and $n$ the column in the direct product.

As a preliminary step we show how $\left((m-2)^{\prime}, n-2\right)$ can be reached. First we reach $\left(4^{\prime}, 4\right)$ by $b a^{2} b$. If $m=6$, then $\left(4^{\prime}, 4\right) a^{q-4}=\left(4^{\prime}, q\right)$ for $q=5, \ldots,(n-2)$. Otherwise, if $m>n$, then $\left(0^{\prime}, 0\right) b a^{3}(b a)^{2}=\left(5^{\prime}, 4\right),\left(5^{\prime}, 4\right)(b a)^{m-n-1}=\left((m-n+4)^{\prime}, 4\right)$, and $(m-n+4,4) a^{n-6}=\left((m-2)^{\prime}, n-2\right)$. If $m=n$, then $\left(4^{\prime}, 4\right) a^{n-6}=\left((m-2)^{\prime}, n-2\right)$. Let $m<n$. Observe that if $(n-m) \equiv p(\bmod 3)$ for $0 \leqslant p \leqslant 2$, then $((3-$ $\left.p)^{\prime}, 4\right) a^{n-m} b a^{m-6}=\left((m-2)^{\prime}, n-2\right)$. Hence we need to reach $\left((3-p)^{\prime}, 4\right)$. We consider the following three cases:

1. $(n-m) \equiv 0(\bmod 3)$.

We reach $\left(3^{\prime}, 4\right)$ by $b^{2} a^{2} b a$, and then apply $a^{n-m} b a^{m-6}$.

2. $(n-m) \equiv 2(\bmod 3)$.

We reach $\left(1^{\prime}, 4\right)$ by $b^{2} a^{2}$, and then apply $a^{n-m} b a^{m-6}$.

3. $(n-m) \equiv 1(\bmod 3)$.

If $m \leqslant n-4$ then $\left(1^{\prime}, 4\right) a^{3} b a^{n-m-3} b a^{m-6}=\left((m-2)^{\prime}, n-2\right)$. Otherwise $m=n-1$. If $n<12$, then we have five cases, where $\left(0^{\prime}, 0\right) b a^{2}=\left(3^{\prime}, 3\right)$ :

(a) $m=6, n=7:\left(4^{\prime}, 4\right) a^{2} b a^{2}=\left(2^{\prime}, 4\right)$.

(b) $m=7, n=8:\left(3^{\prime}, 3\right)(a b)^{2} a^{4} b a^{2}=\left(2^{\prime}, 4\right)$.

(c) $m=8, n=9:\left(3^{\prime}, 3\right) a^{8}=\left(2^{\prime}, 4\right)$.

(d) $m=9, n=10:\left(3^{\prime}, 3\right) a b a^{9}=\left(2^{\prime}, 4\right)$.

(e) $m=10, n=11:\left(3^{\prime}, 3\right) a b a^{4} b a^{3} b a b a^{6} b a^{2}=\left(2^{\prime}, 4\right)$.

Otherwise we reach $\left(2^{\prime}, 4\right)$ as follows: $\left(4^{\prime}, 4\right) a^{m-6}=\left(4^{\prime}, n-2\right),\left(4^{\prime}, n-2\right) a^{m-6}=$ $\left(4^{\prime}, n-7\right),\left(4^{\prime}, n-7\right) b a b a^{2} b a^{3}=\left(2^{\prime}, 1\right),\left(2^{\prime}, 1\right) a^{3}=\left(2^{\prime}, 4\right)$. 
Now, having reached $\left((m-2)^{\prime}, n-2\right)$, we can reach all the remaining pairs: We have $\left((m-2)^{\prime}, n-2\right)(a b)^{2} b=\left((m-1)^{\prime}, 3\right)$, and from $\left((m-1)^{\prime}, 3\right)$ all pairs $\left((m-1)^{\prime}, q\right)$ for $1 \leqslant q \leqslant n-1$ can be reached, since $Q_{n} \backslash\{0, n-1\}$ is a strongly connected component in $\mathcal{D}_{n}$. Similarly, $\left((m-2)^{\prime}, n-2\right) a b^{2}=\left(4^{\prime}, n-1\right)$, and all pairs $\left(p^{\prime}, n-1\right)$ for $1 \leqslant p \leqslant m-1$ can be reached. For the remaining pairs we proceed as follows:

1. Column 3:

(a) $\left((m-2)^{\prime}, n-2\right) a b a=\left(1^{\prime}, 3\right)$,

(b) $(0,0) b a^{3} b=\left(2^{\prime}, 3\right)$,

(c) $(0,0) b a^{2}=\left(3^{\prime}, 3\right)$,

(d) $\left(2^{\prime}, 3\right) a b=\left(4^{\prime}, 3\right)$,

(e) $\left((p-1)^{\prime}, 3\right) a b=\left(p^{\prime}, 3\right)$ for $p=5, \ldots, m-2$.

2. Column 4:

(a) $\left(3^{\prime}, 3\right) a=\left(1^{\prime}, 4\right)$,

(b) $\left(3^{\prime}, 3\right) b=\left(4^{\prime}, 4\right)$,

(c) $\left((p-1)^{\prime}, 3\right) a=\left(p^{\prime}, 4\right)$ for $p=2,3,5, \ldots, m-2$.

3. Column $q$ for $q=5, \ldots, n-2$ :

(a) $\left(3^{\prime}, q-1\right) a=\left(1^{\prime}, q\right)$,

(b) $\left(3^{\prime}, q\right) b=\left(4^{\prime}, q\right)$,

(c) $\left((p-1)^{\prime}, q-1\right) a=\left(p^{\prime}, q\right)$ for $p=2,3,5, \ldots, m-2$.

4. Column 1:

(a) $\left(0^{\prime}, 0\right) b=\left(1^{\prime}, 1\right)$,

(b) $\left((m-2)^{\prime}, n-2\right) a=\left(4^{\prime}, 1\right)$.

(c) $\left((p-1)^{\prime}, n-2\right) a=\left(p^{\prime}, 1\right)$ for $p=2,3,5, \ldots, m-2$.

5. Column 2:

(a) $\left(3^{\prime}, 1\right) a=\left(1^{\prime}, 2\right)$,

(b) $\left(0^{\prime}, 0\right) a^{2}=\left(2^{\prime}, 2\right)$,

(c) $\left(3^{\prime}, 1\right) b=\left(4^{\prime}, 2\right)$,

(d) $\left((p-1)^{\prime}, 1\right) a=\left(p^{\prime}, 2\right)$ for $p=3,5, \ldots, m-2$.

As we discussed in Subsection 3.2, for each $x \in \Sigma$ every state $q \in Q_{n} \backslash\{0, n-1\}$ has a unique predecessor state $p \in Q_{n} \backslash\{n-1\}$. It follows that if $q w=p \in Q_{n} \backslash\{0, n-1\}$, for some state $q$ and word $w$, then $r w \neq p$ for $r \neq q$. The same facts apply to $Q_{m}^{\prime}$. We shall need the following two claims:

Claim 1. From any pair $\left(q^{\prime}, p\right)$ with $1 \leqslant q \leqslant m-2,1 \leqslant p \leqslant n-2$ we can reach $\left(1^{\prime}, 1\right)$.

First we find a word $w$ such that $q^{\prime} w \in\left\{1^{\prime}, 2^{\prime}, 3^{\prime}\right\}$ and $p w=1$. Note that it is sufficient to find $u$ such that $q^{\prime} u \in\left\{1^{\prime}, 2^{\prime}, 3^{\prime}\right\}$ and $p u \in Q_{n} \backslash\{0, n-1\}$, because then 
$w=u a^{n-1-(p u)}$ does the job. So if $q^{\prime} \in\left\{1^{\prime}, 2^{\prime}, 3^{\prime}\right\}$, then we are done. Otherwise use $a^{m-1-q}$, which maps $q^{\prime}$ to $4^{\prime}$. If $p a^{m-1-q} \neq 2$, then we use $b$ to map $4^{\prime}$ to $3^{\prime}$ and keep the state of $\mathcal{D}_{n}$ in $Q_{n} \backslash\{0, n-1\}$, and again we are done. Suppose $p a^{m-1-q}=2$. If $m=6$ then $4^{\prime} a b=3^{\prime}$ and $p a^{m-1-q} a b \neq 2$, and we are done. Otherwise $4^{\prime} a^{m-5}=4^{\prime}$, and also $4^{\prime} a b a^{m-6}=4^{\prime}$. If $2 a^{m-5} \neq 2$ then we can apply an additional $b$ and be done. If $2 a^{m-5}=2$, then $2 a b a^{m-6}=3$, and again by applying an additional $b$ we are done.

Now let $q^{\prime} \in\left\{2^{\prime}, 3^{\prime}\right\}$. We show that there is a word $w$ such that $q^{\prime} w=1$ and $1 w=1$. If $q^{\prime} a^{n-2}=1^{\prime}$ then we are done, and if $q^{\prime} a^{n-2} \in\left\{2^{\prime}, 3^{\prime}\right\} \backslash\left\{q^{\prime}\right\}$, then $q^{\prime}\left(a^{n-2}\right)^{2}=1$ and we have reached $\left(1^{\prime}, 1\right)$. So assume $q^{\prime} a^{n-2}=q^{\prime}$; thus $(n-2) \equiv 0$ mod 3 , and so $n \geqslant 8$.

If $q^{\prime}=3^{\prime}$, then $\left(3^{\prime}, 1\right) a^{4} b a^{n-1-4}=\left(r^{\prime}, 1\right)$ with $r^{\prime} \neq 3^{\prime}$, so we are done by $\left(a^{4} b a^{n-1-4}\right)^{2}$. If $q^{\prime}=2^{\prime}$, then $\left(2^{\prime}, 1\right) a^{5} b a^{n-1-5}=\left(r^{\prime}, 1\right)$ with $r^{\prime} \neq 2^{\prime}$, so we are done by $a^{5} b\left(a^{n-1-5}\right)^{2}$.

Claim 2. For any $q^{\prime} \in Q_{m}^{\prime} \backslash\left\{0^{\prime},(m-1)^{\prime}\right\}$ and $p \in Q_{n} \backslash\{0,(n-1)\}$ there is a word such that $q^{\prime} w \in Q_{m}^{\prime} \backslash\left\{0^{\prime},(m-1)^{\prime}\right\}$ and $p w=n-1$. It follows that $\left(q^{\prime}, p\right)$ can be mapped to $\left(r^{\prime}, n-1\right)$, for any state $r^{\prime} \in Q_{m}^{\prime} \backslash\left\{0^{\prime}\right\}$.

If $q^{\prime} \in\left\{1^{\prime}, 2^{\prime}, 3^{\prime}\right\}$ then let $w_{1}=a^{2} b, a b$, or $b$, respectively, and $\varepsilon$ otherwise; so $w_{1}$ maps $q^{\prime}$ to $\left\{4^{\prime}, \ldots,(m-2)^{\prime}\right\}$. Then let $w_{2}=a^{n-\left(p w_{1}\right)}$; so $w_{2}$ maps $p w_{1}$ to 2 , and $q w_{1}$ is still in $\left\{4^{\prime}, \ldots,(m-2)^{\prime}\right\}$. Then $w=w_{1} w_{2} b$ satisfies the claim. Since $Q_{m}^{\prime} \backslash\left\{0^{\prime},(m-1)^{\prime}\right\}$ is strongly connected, we can map $\left(q^{\prime} w, n-1\right)$ to any $r^{\prime} \in Q_{m}^{\prime} \backslash\left\{0^{\prime}\right\}$.

Now we consider the four cases of the operations. In all cases pair $\left(0^{\prime}, 0\right)$ is distinguished as the only non-empty state which does not accept any word starting with $a$.

1. Union and symmetric difference.

Consider $\left(q_{1}^{\prime}, p_{1}\right)$ and $\left(q_{2}^{\prime}, p_{2}\right)$ with $q_{1}^{\prime} \neq q_{2}^{\prime}$, where $1 \leqslant q_{1}, q_{2} \leqslant m-1$ and $1 \leqslant p_{1}, p_{2} \leqslant n-1$. Without loss of generality, $q_{1}^{\prime} \neq(m-1)^{\prime}$. The same arguments here work for both operations, since we are not mapping the pairs to $\left(1^{\prime}, 1\right)$. By Claim 2 we can map $\left(q_{1}^{\prime}, p_{1}\right)$ to $(1, n-1)$ by some word $w$. Then $q_{2}^{\prime} w \neq 1$. If also $p_{2} \neq 1$, then $\left(q_{2}^{\prime}, p_{2}\right)$ is not final and $w$ distinguishes our pairs. Otherwise we apply Claim 2 once more for $\left(1, p_{2}\right)$, obtaining a word $u$ such that $(1, n-1) u=(1, n-1)$, and $\left(q_{2}^{\prime}, 1\right) u=\left(q_{2}^{\prime} u, n-1\right)$. Since $1 u=u$, we have $q_{2}^{\prime} u \neq 1$, and so $w^{\prime} u$ distinguishes our pairs.

Assume now $q_{1}^{\prime}=q_{2}^{\prime}$ and $p_{1} \neq p_{2}$, where $p_{1} \neq n-1$. Let $w$ be a word mapping $p_{1}$ to 1 ; then $p_{2} w \neq 1$. If $q_{1}^{\prime} w \neq 1^{\prime},\left(q_{2}^{\prime}, p_{2}\right) w=\left(q_{1}^{\prime}, p_{2}\right) w$ is not final so $w$ distinguishes our pairs. Otherwise $w a^{3} b^{2}$ maps $q_{1}^{\prime}$ to $(m-1)^{\prime}$, and $p_{1}$ to 4 . Thus $w a^{3} b^{2} a^{n-6}$ maps $p_{1}$ to 1 , and since $p_{2}$ is mapped elsewhere and $q_{1}^{\prime}=q_{2}^{\prime}$ to $(m-1)^{\prime}$, our pairs are distinguished.

2. Intersection. 
Consider $\left(q_{1}^{\prime}, p_{1}\right)$ and $\left(q_{2}^{\prime}, p_{2}\right)$ with $q_{1}^{\prime} \neq q_{2}^{\prime}$ or $p_{1} \neq p_{2}$, where $1 \leqslant q_{1}, q_{2} \leqslant m-2$ and $1 \leqslant p_{1}, p_{2} \leqslant n-2$. By Claim 1 we can map $\left(q_{1}^{\prime}, p_{1}\right)$ to the final state $\left(1^{\prime}, 1\right)$ by some word $w$. Then either $q_{2}^{\prime} w \neq 1^{\prime}$ or $p_{2} w \neq 1,\left(q_{2}^{\prime}, p_{2}\right)$ is not final and our pairs are distinguished. Together with $\left(0^{\prime}, 0\right)$ and $\left((m-1)^{\prime}, n-1\right)$ these give $2+(m-2)(n-2)=m n-2(m+n-3)$ distinguished pairs.

3. Difference.

Consider $\left(q_{1}^{\prime}, p_{1}\right)$ and $\left(q_{2}^{\prime}, p_{2}\right)$ with $q_{1}^{\prime} \neq q_{2}^{\prime}$, where $1 \leqslant q_{1}, q_{2} \leqslant m-2$ and $1 \leqslant p_{1}, p_{2} \leqslant n-1$. This follows in exactly the same way as the corresponding case of union and symmetric difference. Assume now $q_{1}^{\prime}=q_{2}^{\prime}$ and $p_{1} \neq p_{2}$. Without loss of generality, $p_{1} \neq n-1$. By Claim 1 we can map $\left(q_{1}^{\prime}, p_{1}\right)$ to the non- final state $\left(1^{\prime}, 1\right)$ by some word $w$. Then $\left(q_{2}^{\prime}, p_{2}\right) w=\left(1^{\prime}, p_{2} w\right)$, and since $p_{2} w \neq p_{1} w=1,\left(1^{\prime}, p_{2} w\right)$ a final state. Thus $w$ distinguishes our pairs.

Together with $\left(0^{\prime}, 0\right)$ and $\left((m-1)^{\prime}, n-1\right)$ these give $2+(m-2)(n-1)=$ $m n-(m+2 n-4)$ distinguished pairs.

\section{Witnesses with Semigroups in $T^{\geqslant 6}(n)$}

We now turn to the operations which cannot have witnesses with transition semigroups in $\mathbf{T}^{\leqslant 5}$.

Definition 2. For $n \geqslant 4$, we define the DFA $\mathcal{D}_{n}(a, b, c, d, e)=\left(Q_{n}, \Sigma, \delta, 0, F\right)$, where $Q_{n}=\{0, \ldots, n-1\}, \Sigma=\{a, b, c, d, e\}, \delta$ is defined by the transformations

- $a:(0 \rightarrow n-1)(1, \ldots, n-2)$,

- $b:(0 \rightarrow n-1)(1,2)$,

- $c:(0 \rightarrow n-1)(n-2 \rightarrow 1)$,

- $d:(\{0,1\} \rightarrow n-1)$,

- $e:(Q \backslash\{0\} \rightarrow n-1)(0 \rightarrow 1)$,

and $F=\left\{q \in Q_{n} \backslash\{0, n-1\} \mid q\right.$ is odd $\}$. For $n=4$, $a$ and $b$ coincide, and we can use $\Sigma=\{b, c, d, e\}$. The structure of $\mathcal{D}_{5}(a, b, c, d, e)$ is illustrated in Figure 3.

Our main result in this section is the following theorem: 
Theorem 6 (Boolean Operations, Reversal, Number and Complexity of Atoms, Syntactic Complexity). Let $\mathcal{D}_{n}(a, b, c, d, e)$ be the DFA of Definition 2, and let the language it accepts be $L_{n}(a, b, c, d, e)$. Then $L_{n}(a, b, c, d, e)$ meets the bounds for Boolean operations, reversal, number and quotient complexity of atoms, and syntactic complexity as follows:

1. For $n, m \geqslant 4, L_{m}(a, b,-, d, e)$ and $L_{n}(b, a,-, d, e)$ meet the bounds $m n-(m+$ $n-2)$ for union and symmetric difference, $m n-2(m+n-3)$ for intersection, and $m n-(m+2 n-4)$ for difference.

2. For $n \geqslant 4, L_{n}(a,-, c,-, e)$ meets the bound $2^{n-2}+1$ for reversal and number of atoms.

3. For $n \geqslant 6, L_{m}(a, b, c, d, e)$ meets the bound $(n-1)^{n-2}+n-2$ for syntactic complexity, and the bounds on the quotient complexities of atoms.

The claim about syntactic complexity is known from [20]. It was shown in [21] that the number of atoms of a regular language $L$ is equal to the quotient complexity of $L^{R}$. In the next subsections we prove the claim about Boolean operations, reversal, and atom complexity. First we state some properties of $\mathcal{D}_{n}$.

Proposition 6. For $n \geqslant 4$ the DFA of Definition 2 is minimal, accepts a suffix-free language, and its transition semigroup $T_{n}$ has cardinality $(n-1)^{n-2}+n-2$. In particular, $T_{n}$ contains (a) all $(n-1)^{n-2}$ transformations that send 0 and $n-1$ to $n-1$ and $\operatorname{map} Q_{n} \backslash\{0, n-1\}$ to $Q_{n} \backslash\{0\}$, and (b) all $n-2$ transformations that send 0 to a state in $Q_{n} \backslash\{0, n-1\}$ and map all the other states to $n-1$. Also, $T_{n}$ is generated by $\{a, b, c, d, e\}$ and cannot be generated by a smaller set of transformations.

Proof. To prove minimality, note first that only the initial state 0 accepts $e$, and only state $n-1$ accepts nothing. Suppose $p, q \in Q_{n} \backslash\{0, n-1\}, p<q$, and $p$ and $q$ have the same parity; then $p a^{n-2-p} c=1$, which is a final state, but $q a^{n-2-q} c$ is an even state, which is non-final. If $p$ and $q$ have different parities, then one is final while the other is not. Hence $\mathcal{D}_{n}$ is minimal.

The language $L_{n}$ is suffix-free, because every word in $L_{n}$ has the form $e x$ with $x \in\{a, b, c, d\}$. The claims about cardinality of $T_{n}$, transformations, and generators were proved in $[12,20]$.

\subsection{Boolean Operations}

We now show that witness DFAs for Boolean operations may have transition semigroups in $\mathbf{T} \geqslant 6$. Thus Boolean operations have witnesses with transition semigroups that are subsemigroups of either $\mathbf{T}^{\leqslant 5}$ or $\mathbf{T}^{\geqslant 6}$. This is sharp contrast with all the other complexity measures: any witness for star and any second witness for 
product can only be associated with $\mathbf{T}^{\leqslant 5}$, while the size of the syntactic semigroup, reversal, and complexities of atoms must have witnesses associated with $\mathbf{T}^{\geqslant 6}$.

Theorem 7. For $n, m \geqslant 4, L_{m}(a, b,-, d, e)$ and $L_{n}(b, a,-, d, e)$ meet the bounds $m n-(m+n-2)$ for union and symmetric difference, $m n-2(m+n-3)$ for intersection, and $m n-(m+2 n-4)$ for difference.

Proof. Our two DFAs $\mathcal{D}_{m}(a, b,-, d, e)$ and $\mathcal{D}_{n}(b, a,-, d, e)$ are illustrated in Figure 6; consider their direct product. Let $H=\left\{\left((m-1)^{\prime}, q\right) \mid 1 \leqslant q \leqslant n-2\right\}, V=$ $\left\{\left(p^{\prime}, n-1\right) \mid 1 \leqslant p \leqslant m-2\right\}$, and $M=\left\{\left(p^{\prime}, q\right) \mid 1 \leqslant p \leqslant m-2,1 \leqslant q \leqslant n-2\right\}$.

Let $\mathcal{A}_{n}(a, b)=\left(Q_{n} \backslash\{0, n-1\},\{a, b\}, \delta, 1, F\right)$ be the DFA obtained from the DFA $\mathcal{D}_{n}(a, b,-, d, e)$ by restricting the alphabet to $\{a, b\}$ and the set of states to $\{1, \ldots, n-2\}$. Since DFAs $\mathcal{A}_{m}^{\prime}(a, b)$ and $\mathcal{A}_{n}(b, a)$ have ordered pairs $\left\{\delta_{a}^{\prime}, \delta_{b}^{\prime}\right\}$ and $\left\{\delta_{a}, \delta_{b}\right\}$ of transformations that generate the symmetric groups of degrees $m$ and $n$ and are not conjugate, the result from [2, Theorem 1] applies, except in our case where $m=4$ and $n=4$ (we add two states to the $m$ and $n$ in [2]). We have verified this case by computation. Therefore we know that all states in $M$ are reachable from state $\left(1^{\prime}, 1\right)$ and all pairs of such states are distinguishable.

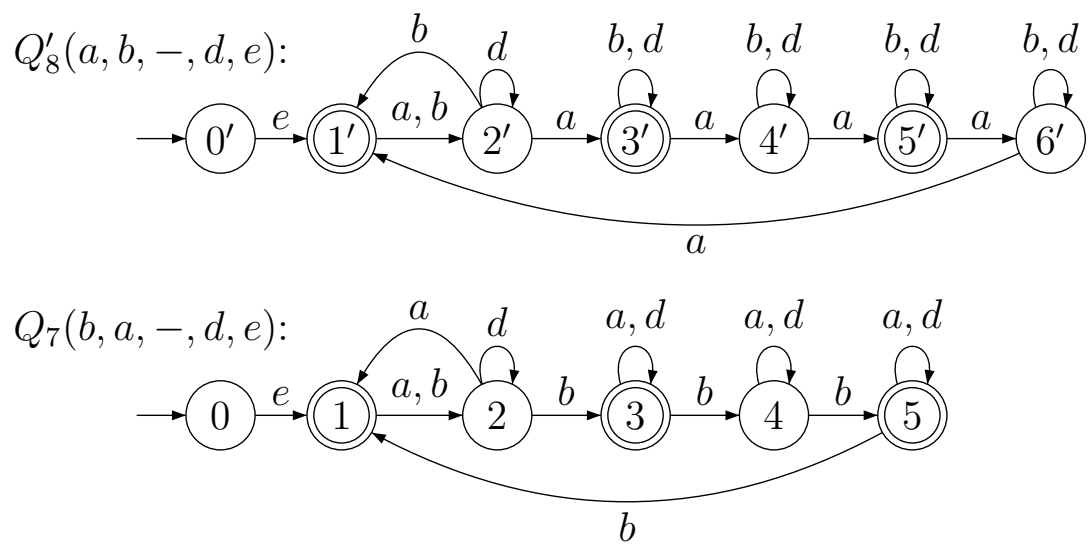

Figure 6: The DFAs $\mathcal{D}_{8}^{\prime}(a, b,-, d, e)$ and $\mathcal{D}_{7}(b, a,-, d, e)$ for Boolean operations. The empty states $8^{\prime}$ and 7 and the transitions to them are omitted.

We show that $\left(0^{\prime}, 0\right),\left((m-1)^{\prime}, n-1\right)$ and all states in $H, V$ and $M$ are reachable. State $\left(0^{\prime}, 0\right)$ is the initial state, $\left(1^{\prime}, 1\right)$ is reached from $\left(0^{\prime}, 0\right)$ by $e$ and $\left((m-1)^{\prime}, n-1\right)$ by $a$. By Theorem 1 of [2] all the states in $M$, and in particular $\left(1^{\prime}, 2\right)$ and $\left(2^{\prime}, 1\right)$, are reachable from $\left(1^{\prime}, 1\right)$. From state $\left(1^{\prime}, 2\right)$ we reach $\left((m-1)^{\prime}, 2\right)$ by $d$ and from there, state $\left((m-1)^{\prime}, q\right)$ by a word in $b^{*}$ for $1 \leqslant q \leqslant n-2$. Symmetrically, from $\left(2^{\prime}, 1\right)$ we 
reach $\left(2^{\prime}, n-1\right)$, and from there, state $\left(p^{\prime}, n-1\right)$ for $1 \leqslant p \leqslant m-2$ by a word in $a^{*}$. Hence all $(m-1)(n-1)+1=m n-(m+n-2)$ states are reachable.

State $\left(0^{\prime}, 0\right)$ is the only state accepting a word beginning with $e$. State $((m-$ $\left.1)^{\prime}, n-1\right)$ is the only empty state.

For union we consider six possibilities for the distinguishability of two states:

1. States in $H$. If $n$ is odd, and $p^{\prime}, r^{\prime} \in Q_{n}^{\prime} \backslash\left\{0^{\prime},(n-1)^{\prime}\right\}$ with $p<r$, where $p^{\prime}$ and $r^{\prime}$ are both final or both non-final, then $r^{\prime}$ accepts $a^{n-1-r}$ whereas $p^{\prime}$ rejects it. If $n$ is even, $r^{\prime}$ accepts $a^{n-r} b$ whereas $p^{\prime}$ rejects it. Hence if $\left((m-1)^{\prime}, p\right)$ and $\left((m-1)^{\prime}, r\right)$ are in $H$, they are distinguishable.

2. States in $V$. The argument is symmetric to that in $H$.

3. $H$ and $V$. Consider $\left(p^{\prime}, n-1\right) \in V$ and $\left((m-1)^{\prime}, q\right) \in H$. Note that $\left(1^{\prime}, n-1\right)$ accepts $a^{2} b$ whereas $\left((m-1)^{\prime}, 1\right)$ rejects it. For every $\left(p^{\prime}, q\right)$ there exists a permutation $t$ induced by a word $w$ in $\{a, b\}^{*}$ such that $\left(1^{\prime}, 1\right) t=\left(p^{\prime}, q\right)$. Then $\left(p^{\prime}, q\right) t^{-1}=\left(1^{\prime}, 1\right)$. Hence $\left(p^{\prime}, n-1\right)$ and $\left((m-1)^{\prime}, q\right)$ are mapped by $t^{-1}$ to $\left(1^{\prime}, n-1\right)$ and $\left((m-1)^{\prime}, 1\right)$, which are distinguishable.

4. $H$ and $M$. If $\left(p^{\prime}, r\right) \in M$, then there exists a permutation $t$ induced by a word $w$ in $\{a, b\}^{*}$ such that $\left(p^{\prime}, r\right) t=\left(1^{\prime}, 2\right)$. The same $t$ maps $\left((m-1)^{\prime}, q\right) \in H$ to some $\left((m-1)^{\prime}, s\right)$ with $1 \leqslant s \leqslant n-2$. Then $\left(1^{\prime}, 2\right) d=\left((m-1)^{\prime}, 2\right)$. If $s \neq 1$, then $s d \in\{2, \ldots, n-2)$, and we are done, since states $\left((m-1)^{\prime}, 2\right)$ and $\left((m-1)^{\prime}, s\right)$ are in $H$ and hence are distinguishable. Otherwise, $\left((m-1)^{\prime}, s\right) d=\left((m-1)^{\prime}, n-1\right)$ and again we are done.

5. $V$ and $M$. The argument is symmetric to that for $H$ and $M$.

6. States in $M$. Apply Theorem 1 of [2].

For symmetric difference, we have as many states as for union. The arguments for distinguishability are exactly the same as for union.

For intersection, all states in $H \cup V \cup\left\{\left((m-1)^{\prime}, n-1\right)\right\}$ are empty, and all states in $M$ are distinguishable by [2, Theorem 1]. Hence there are $(m-2)(n-2)+2=$ $m n-2(m+n-3)$ states altogether.

For difference, all states in $H \cup\left\{\left((m-1)^{\prime}, n-1\right)\right\}$ are empty, so this leaves $(m-2)(n-1)+2=m n-(m+2 n-4)$ states. The states in $V$ are all distinct by the argument used for union. Also $\left(p^{\prime}, q\right) \in M$ is distinguishable from $\left(r^{\prime}, n-1\right) \in V$, by the argument used for union.

Since $\delta_{d}=\delta_{c_{1}} \delta_{c_{1}}$ and $\delta_{e}=\delta_{c_{1}} \delta_{c_{2}} \cdots \delta_{c_{n-1}}$, where the $c_{i}$ are from Proposition 2, the semigroup of $\mathcal{D}_{n}(a, b,-, d, e)$ is in $\mathbf{T}^{\leqslant 5}(n) \cap \mathbf{T}^{\geqslant 6}(n)$. In fact, one can verify that the semigroup of $\mathcal{D}_{n}(a, b,-, d, e)$ is $\mathbf{T}^{\leqslant 5}(n) \cap \mathbf{T}^{\geqslant 6}(n)$. 


\subsection{Reversal}

Han and Salomaa [26] showed that to meet the bound for reversal one can use the binary DFA of Leiss [32] and add a third input to get a suffix-free DFA. Cmorik and Jirásková [23] showed that a binary alphabet will not suffice. We show a different ternary witness below, and prove that any witness must have its transition semigroup in $T^{\geqslant 6}$.

Theorem 8 (Semigroup of Reversal Witness). For $n \geqslant 4$, the transition semigroup of a minimal DFA $\mathcal{D}_{n}\left(Q_{n}, \Sigma, \delta, 0, F\right)$ of a suffix-free language $L_{n}$ that meets the bound $2^{n-2}+1$ for reversal is a subsemigroup of $\mathbf{T}^{\geqslant 6}(n)$.

Proof. It suffices to show that no pair of states is colliding.

We construct an NFA $\mathcal{N}$ for $L_{n}^{R}$ by reversing the transitions of $\mathcal{D}$, and interchanging the sets of final and initial states. We then determinize $\mathcal{N}$ using the subset construction to get a DFA $\mathcal{R}$ for $L_{n}^{R}$. The states of $\mathcal{R}$ are sets of states of $\mathcal{D}$.

Consider a reachable subset $S \subseteq Q_{n}$. We can assume that $n-1 \notin S$, since $n-1$ is not reachable from a start state in $\mathcal{N}$. Also, if $0 \in S$ then $S=\{0\}$, as otherwise in $\mathcal{D}$ some transformation would map both 0 and $q \in\{1, \ldots, n-1\}$ to a final state so the language would not be suffix-free [26, Lemma 6]. Hence, every subset $S \subseteq\{1, \ldots, n-2\}$ must be reachable.

Suppose that there are two distinct states $p, q \in\{1, \ldots, n-2\}$ such that the pair $\{p, q\}$ is colliding. This means that $0 \delta_{w}=p$ and $q^{\prime} \delta_{w}=q$ for some word $w$ and $q^{\prime} \in\{1, \ldots, n-2\}$. Since the subset $\{p, q\}$ is reachable in $T_{\mathcal{D}^{R}}$, there is some $\left(\delta_{u}\right)^{-1}$ such that $F\left(\delta_{u}\right)^{-1}=\{p, q\}$. So $p \delta_{u} \in F$ and $q\left(\delta_{u}\right) \in F$. But then $w u \in L_{n}$, and also $v w u \in L_{u}$, where $v$ is such that $0 \delta_{v}=q^{\prime}$, which contradicts that $L_{n}$ is suffix-free.

Theorem 9 (Reversal Complexity). If $n \geqslant 4$, then $L_{n}(a,-, c,-, e)$ of Definition 2 meets the bound $2^{n-2}+1$ for reversal.

Proof. As before, we construct NFA $\mathcal{N}$ for $L^{R}$ - state $n-1$ is now not reachable and determinize $\mathcal{N}$ to get the DFA $\mathcal{R}$. We shall show that we can reach $\{0\}$ and all subsets of $Q_{n} \backslash\{0, n-1\}$.

The initial state of $\mathcal{N}$ consists of all the odd states in $Q_{n} \backslash\{0, n-1\}$. By applying $e$ we reach the set $\{0\}$. By applying $e$ again, we reach $\emptyset$. To reach $Q_{n} \backslash\{0, n-1\}$ from $F$ we apply $c\left(a^{2} c\right)^{\lceil n / 2\rceil-1}$.

Consider a non-empty proper subset $S \subsetneq Q_{n} \backslash\{0, n-1\}$. Since $a$ acts cyclically on $Q_{n} \backslash\{0, n-1\}$, it is sufficient to prove reachability for $S$ that contains $n-2$ if $n$ is odd, and does not contain $n-2$ if $n$ is even; thus $S$ agrees with $F$ on $n-2$. We start from $F=\{1,3, \ldots, n-4, n-2\}$ and transform it inductively. Assume that for $i \geqslant 0$, we have reached $F_{i}$ which agrees with $S$ on states $1, \ldots, i$ and with $F$ on states 
$i+1, \ldots, n-2$. Of course, $F_{0}=F$. If $S$ and $F_{i}$ agree on $i+1$, then we already have $F_{i+1}=F_{i}$. Otherwise $i+1 \in F_{i}$ if and only if $i+1 \notin S$. We have $i+1<n-2$, since we know that $S$ agrees with $F$ on $n-2$. Then $i+1 \in F_{i}$ if and only if $i+2 \notin F_{i}$. We apply $a^{i+1} c a^{n-3-i}$ to $F_{i}$, which yields the subset $F_{i+1}$ differing from $F_{i}$ only in state $i+1$; thus $i+1 \in F_{i+1}$ if and only if $i+1 \in S$. The induction follows, and so $S=F_{n-2}$ is reachable.

Now we prove that these $2^{n-2}+1$ states (sets of states of $\mathcal{D}$ ) are pairwise distinguishable. Set $\{0\}$ is the only final state. Consider two sets $R, S \subseteq Q_{n} \backslash\{0, n-1\}$ and suppose that $q \in R \oplus S$. Then $a^{q-1} e$ is accepted from the set that contains $q$ but not from other set. Therefore there are no equivalent states.

Although $L_{n}(a,-, c,-, e)$ meets the bound for the number of atoms, it does not meet the bounds on the quotient complexity of atoms.

\subsection{Complexity of Atoms in Suffix-Free Languages}

Let $Q_{n}=\{0, \ldots, n-1\}$ and let $L$ be a non-empty regular language with quotient set $K=\left\{K_{0}, \ldots, K_{n-1}\right\}$. Let $\mathcal{D}=\left(Q_{n}, \Sigma, \delta, 0, F\right)$ be the minimal DFA of $L$ in which the language of state $q$ is $K_{q}$.

Denote the complement of a language $L$ by $\bar{L}=\Sigma^{*} \backslash L$. Each subset $S$ of $Q_{n}$ defines an atomic intersection $A_{S}=\bigcap_{i \in S} K_{i} \cap \bigcap_{i \in \bar{S}} \overline{K_{i}}$, where $\bar{S}=Q_{n} \backslash S$. An atom of $L$ is a non-empty atomic intersection. Since atoms are pairwise disjoint, every atom $A$ has a unique atomic intersection associated with it, and this atomic intersection has a unique subset $S$ of $K$ associated with it. This set $S$ is called the basis of $A$.

Let $A_{S}=\bigcap_{i \in S} K_{i} \cap \bigcap_{i \in \bar{S}} \overline{K_{i}}$ be an atom. For any $w \in \Sigma^{*}$ we have

$$
w^{-1} A_{S}=\bigcap_{i \in S} w^{-1} K_{i} \cap \bigcap_{i \in \bar{S}} \overline{w^{-1} K_{i}}
$$

Since a quotient of a quotient of $L$ is also a quotient of $L, w^{-1} A_{S}$ has the form:

$$
w^{-1} A_{S}=\bigcap_{i \in X} K_{i} \cap \bigcap_{i \in Y} \overline{K_{i}}
$$

where $|X| \leqslant|S|$ and $|Y| \leqslant n-|S|, X, Y \subseteq Q_{n}$.

Proposition 7. Suppose $L$ is a suffix-free language with $n \geqslant 4$ quotients. Then $L$ has at most $2^{n-2}+1$ atoms. Moreover, the complexity $\kappa\left(A_{S}\right)$ of atom $A_{S}$ satisfies 


$$
\kappa\left(A_{S}\right) \begin{cases}\leqslant 2^{n-2}+1, & \text { if } S=\emptyset ; \\
=n, & \text { if } S=\{0\} ; \\
\leqslant 1+\sum_{x=1}^{|S|} \sum_{y=0}^{n-2-|S|}\left(\begin{array}{c}
n-2 \\
x
\end{array}\right)\left(\begin{array}{c}
n-2-x \\
y
\end{array}\right), & \emptyset \neq S \subseteq\{1, \ldots, n-2\} .\end{cases}
$$

Proof. If $n-1 \in S$, then $A_{S}$ is not an atom. Also, $K_{0} \cap K_{i}=\emptyset$ for $i=1, \ldots, n-1$, since $L$ is suffix-free. Hence $0 \in S$ implies $S=\{0\}$. It follows that there are at most $2^{n-2}+1$ atoms.

For atom complexity, consider the following cases:

1. $S=\emptyset$. Here $\bar{S}=Q_{n}, A_{\emptyset}=\bigcap_{i \in Q_{n}} \overline{K_{i}}$ and for $w \in \Sigma^{+}, w^{-1} A_{S}=\bigcap_{i \in Y} \overline{K_{i}}$, where $0 \notin Y$, since $w^{-1} L=L$ only if $w=\varepsilon$, and $n-1$ is always in $Y$ since $w^{-1} K_{n-1}=K_{n-1}$ because $K_{n-1}=\emptyset$. Thus we have the initial quotient $A_{\emptyset}$ with $0 \in \bar{S}$ and at most $2^{n-2}$ choices for the other quotients of $A_{S}$. Hence the complexity of $A_{\emptyset}$ is at most $2^{n-2}+1$.

2. $S=\{0\}$. Since the language is suffix-free, we have that $K_{0} \cap K_{q}=\emptyset$ for all $q \in Q \backslash\{0\}$. Thus $A_{0}=K_{0} \cap \overline{K_{1}} \cap \ldots \cap \overline{K_{n-1}}=K_{0}$, and $K_{0}=L_{n}$ has $n$ quotients.

3. Since $n-1$ always appears in $\bar{S}$ and the $S=\{0\}$ case is done, there remain the cases where $\emptyset \neq S \subseteq Q_{n} \backslash\{0, n-1\}$. Suppose $(X, Y \cup\{n-1\})$, where $n-1 \notin Y$, represents a non-empty quotient of $A_{S}$ by a non-empty word $w$. Since 0 appears only initially, $Y$ cannot contain 0 . Then $X$ must have at least one and at most $|S|$ elements from $\{1, \ldots, n-2\}$, and $Y \subseteq\{1, \ldots, n-2\} \backslash X$. If $0 \delta_{w}=n-1$, then all the states that are mapped to $Y$ by $\delta_{w}$ are from $\{1, \ldots, n-2\} \backslash S$, so in $Y$ there must be from 0 to $n-2-|S|$ states. Hence, together with the empty quotient, we have the formula from Equation 2. Suppose that $0 \delta_{w} \neq$ $n-1$. The 0 -path in $\delta_{w}$ is aperiodic and ends in $n-1$, so there exists a state $p \in\{1, \ldots, n-2\}$ such that $p \delta_{w}=n-1$. If $p \in S$, then $n-1 \in X$, and so $(X, Y)$ represents the empty quotient. If $p \in\{1, \ldots, n-2\} \backslash S$, then again $Y$ contains at most $n-2-|S|$ states.

Following Iván [29] we define a DFA for each atom:

Definition 3. Suppose $\mathcal{D}=\left(Q_{n}, \Sigma, \delta, 0, F\right)$ is a minimal DFA and let $S \subseteq Q_{n}$. Define the DFA $\mathcal{D}_{S}=\left(Q_{S}, \Sigma, \Delta,(S, \bar{S}), F_{S}\right)$, where

- $Q_{S}=\left\{(X, Y) \mid X, Y \subseteq Q_{n}, X \cap Y=\emptyset\right\} \cup\{\perp\}$. 
- For all $a \in \Sigma,(X, Y) a=(X a, Y a)$ if $X a \cap Y a=\emptyset$, and $(X, Y) a=\perp$ otherwise; and $\perp a=\perp$.

- $F_{S}=\{(X, Y) \mid X \subseteq F, Y \subseteq \bar{F}\}$.

DFA $\mathcal{D}_{S}$ recognizes the atomic intersection $A_{S}$ of $L$. If $\mathcal{D}_{S}$ recognizes a non-empty language, then $A_{S}$ is an atom.

Theorem 10. For $n \geqslant 4$, the language $L_{n}\left(\mathcal{D}_{n}(a, b, c, d, e)\right)$ of Definition 2 meets the bounds of Proposition 7 for the atoms.

Proof. There are three cases to consider:

1. $S=\emptyset$. The initial state of $\mathcal{D}_{S}$ is $\left(\emptyset, Q_{n}\right)$. Since $\left(\emptyset, Q_{n}\right) e d=(\emptyset,\{n-1\})$, which is a final state, $A_{\emptyset}$ is an atom. For any non-empty word $w$, we have a quotient of $A_{\emptyset}$ represented by $(\emptyset, Y)$, where $0 \notin Y, n-1 \in Y$, and $Y \backslash\{n-1\}$ is a subset of $Q_{n} \backslash\{0, n-1\}$. By Proposition 6 the transition semigroup of $\mathcal{D}$ contains all transformations that send 0 and $n-1$ to $n-1$ and map $Q_{n} \backslash\{0, n-1\}$ into $Q_{n} \backslash\{0\}$; hence all $2^{n-2}$ quotients of $A_{\emptyset}$ by non-empty words are reachable. Together with $A_{\emptyset}$ we have $2^{n-2}+1$ quotients of $A_{\emptyset}$.

Since $\left(\emptyset, Q_{n}\right) e=(\emptyset,\{1, n-1\})$, which is non-final, and $(\emptyset, Y) e=(\emptyset,\{n-1\})$, which is final, $(\emptyset, Y)$ is distinguished from the initial state. We claim also that all pairs $(\emptyset, Y)$ and $\left(\emptyset, Y^{\prime}\right), Y \neq Y^{\prime}$, are distinguishable. Without loss of generality, suppose that $q \in Y \backslash Y^{\prime}$. If we map $q$ to 1 and $\left(Y \cup Y^{\prime}\right) \backslash\{1\}$ to $n-1$ by a word $w$, then $(\emptyset, Y) w=(\emptyset,\{1, n-1\})$, which is non-final, and $\left(\emptyset, Y^{\prime}\right) w=(\emptyset,\{n-1\})$, which is final. Hence the bound $2^{n-2}+1$ is met.

2. $S=\{0\}$. The initial state of $\mathcal{D}_{S}$ is $\left(\{0\}, Q_{n} \backslash\{0\}\right)$. We have $\left(\{0\}, Q_{n} \backslash\{0\}\right) x=\perp$ for all $x \in \Sigma \backslash\{e\}$, and $\left(\{0\}, Q_{n} \backslash\{0\}\right) e=(\{1\},\{n-1\})$. From $(\{1\},\{n-1\})$ we can reach any state $(\{q\},\{n-1\})$ with $q \in Q_{n} \backslash\{0, n-1\}$. Thus we can reach $n$ states altogether: the initial state, $n-2$ states $(\{q\},\{n-1\})$ with $q \in Q_{n} \backslash\{0, n-1\}$, and $\perp$.

Since $\left(\{0\}, Q_{n} \backslash\{0\}\right) e=(\{1\},\{n-1\})$, which is final, the initial state is nonempty and so differs from $\perp$. For $q \in Q_{n} \backslash\{0, n-1\}$, consider the word $w$ that maps $q$ to 1 and $Q_{n} \backslash\{0, q\}$ to $\{n-1\}$. Then $(\{q\},\{n-1\}) w=(\{1\},\{n-1\})$, which is final, while $\left(\{0\}, Q_{n} \backslash\{0\}\right) w=(\{n-1\}, Y)$ (since $w$ does not contain $e)$, which is non-final since $Y$ contains 1 . Hence $(\{q\},\{n-1\})$ is distinguishable from the initial state and $\perp$. Finally, for $p, q \in Q_{n} \backslash\{0, n-1\},(\{p\},\{n-1\})$ is distinguishable from $(\{q\},\{n-1\})$ by the mapping that sends $p$ to 1 and $q$ to $n-1$. Hence the bound $n$ is met. 
3. $\emptyset \neq S \subseteq Q_{n} \backslash\{0, n-1\}$. The initial state here is $(S, \bar{S})$, where $\emptyset \neq S \subseteq$ $\{1, \ldots, n-2\}$. Since we can map $S$ to $\{1\}$ and $\bar{S}$ to $\{n-1\}$, each such intersection is an atom. If $M=\{1, \ldots, n-2\}$, the atom has the form $(S, \bar{S})=$ $(S,(M \backslash S) \cup\{0, n-1\})$. By applying $e$, we get $\perp$. To get a state of $A_{S}$ different from $\perp$, we can map $S$ to any non-empty subset $X$ of $M$ of cardinality $|X|$, where $1 \leqslant|X| \leqslant|S|$; we can map $M \backslash S$ to any subset $Y$ of $(M \cup\{n-1\}) \backslash X$ of cardinality $|Y|$, where $0 \leqslant|Y| \leqslant|M|-|X|$; and we can map 0 and $n-1$ to $n-1$. Each such intersection $(X, Y \cup\{n-1\})$ represents a non-empty quotient of $A_{S}$ by a non-empty word because we can map $X$ to $\{1\}$ and $Y$ to $\{n-1\}$. Hence we have the formula in Equation 2 for the number of reachable states of $\mathcal{D}_{S}$.

Now consider two states $(X, Y \cup\{n-1\})$ and $\left(X^{\prime}, Y^{\prime} \cup\{n-1\}\right)$, where one of these two states could be the initial one. If $X \neq X^{\prime}$, assume that $q \in X^{\prime} \backslash X$. Let $w$ map $X$ to $\{1\}$ and $Q_{n} \backslash X$ to $n-1$. Then $(X, Y \cup\{n-1\}) w=(\{1\},\{n-1\})$, while the first component of $\left(X^{\prime}, Y^{\prime} \cup\{n-1\}\right) w$ contains $n-1$, meaning that that state is $\perp$; so these states are distinguishable. A symmetric argument applies if $q \in X \backslash X^{\prime}$.

This leaves the case where $X=X^{\prime}$. Then $X$ is disjoint from both $Y$ and $Y^{\prime}$. If $q \in Y^{\prime} \backslash Y$, let $w \operatorname{map} X$ to $\{1\}, Y \cup\{0\}$ to $\{n-1\}$, and $Y^{\prime} \backslash Y$ to $\{1\}$. Then $(X, Y \cup\{n-1\}) w=(\{1\},\{n-1\})$, while $\left(X, Y^{\prime} \cup\{n-1\}\right) w=(\{1\},\{1, n-$ $1\})=\perp$, and we have distinguishablity. A symmetric argument applies if $q \in Y \backslash Y^{\prime}$.

Theorem 11. The transition semigroup $T_{n}$ of a minimal DFA $\mathcal{D}_{n}\left(Q_{n}, \Sigma, \delta, 0, F\right)$ with $n \geqslant 4$ of a suffix-free language $L_{n}$ that meets the bounds for atom complexities from Proposition 7 is a subsemigroup of $\mathbf{T}^{\geqslant 6}(n)$ and is not a subsemigroup of $\mathbf{T}^{\leqslant 5}(n)$.

Proof. It is sufficient to show that every pair of states $p, q \in\{1, \ldots, n-2\}$ is focused by some transformation from $T_{n}$.

Let $p, q \in\{1, \ldots, n-2\}$ be two distinct states. Consider the atom $A_{\{p, q\}}$. From the proof of Proposition 7, to meet the bound for the quotient complexity of $A_{\{p, q\}}$ every pair $(X, Y \cup\{n-1\})$ must represent a quotient of the atom by a non-empty word $w$, where $X, Y \subseteq\{1, \ldots, n-2\}$ are disjoint, $1 \leqslant|X| \leqslant 2$, and $|Y| \leqslant n-2-|X|$. In particular, when $|X|=1$ we know that the transformation of $w$ maps both $p$ and $q$ to the state from $X$, and so $p$ and $q$ are focused.

Tables 1 and 2 show the quotient complexities of atoms for small $n$. 
Table 1: Suffix-free atom complexity. The entries from left to right are suffix-free language, left ideal, regular language. The $*$ stands for "not applicable".

\begin{tabular}{|c|c|c|c|c|c|c|}
\hline$n$ & 1 & 2 & 3 & 4 & 5 & $\ldots$ \\
\hline \hline$|S|=0$ & $* / \mathbf{1} / \mathbf{1}$ & $* / \mathbf{2} / \mathbf{3}$ & $* / 4 / 7$ & $5 / 8 / 15$ & $9 / 16 / 31$ & $\ldots$ \\
\hline \hline$|S|=1$ & $* / \mathbf{1} \mathbf{1}$ & $* / \mathbf{2} / \mathbf{3}$ & $* / \mathbf{5 / 1 0}$ & $\mathbf{5 / 1 3 / 2 9}$ & $13 / 33 / 76$ & $\ldots$ \\
\hline$|S|=2$ & & $* / \mathbf{2} / \mathbf{3}$ & $* / 4 / \mathbf{1 0}$ & $4 / \mathbf{1 6} / \mathbf{4 3}$ & $\mathbf{1 6 / 5 3 / 1 4 1}$ & $\ldots$ \\
\hline$|S|=3$ & & & $* / 3 / 7$ & $* / 8 / 29$ & $8 / 43 / \mathbf{1 4 1}$ & $\cdots$ \\
\hline$|S|=4$ & & & & $* / 4 / 15$ & $* / 16 / 76$ & $\cdots$ \\
\hline$|S|=5$ & & & & & $* / 5 / 31$ & $\cdots$ \\
\hline max & $* / 1 / 1$ & $* / 2 / 3$ & $* / 5 / 10$ & $5 / 16 / 43$ & $16 / 53 / 141$ & $\ldots$ \\
\hline ratio & - & $* / 2.00 / 3.00$ & $* / 2.50 / 3.33$ & $* / 3.20 / 4.30$ & $3.20 / 3.31 / 3.28$ & $\ldots$ \\
\hline
\end{tabular}

\section{Conclusions}

It may appear that the semigroup $\mathbf{T}^{\leqslant 5}(n)$ should not be of great importance, since it exceeds $\mathbf{T}^{\geqslant 6}(n)$ only for $n=4$ and $n=5$, and therefore should not matter when $n$ is large. However, our results show that this is not the case. We conclude with our result about the non-existence of single universal suffix-free witness.

Theorem 12. For $n \geqslant 4$, there does not exist a most complex suffix-free language.

Proof. If there exists a most complex DFA $\mathcal{D}\left(Q_{n}, \Sigma, \delta, 0, F\right)$, then in particular it would have to meet the bound for reversal and star. From Theorem 8 the transition semigroup of $\mathcal{D}$ must be a subsemigroup of $\mathbf{T}^{\geqslant 6}(n)$, but from Theorem 2 we know that it cannot be a subsemigroup of $\mathbf{T}^{\geqslant 6}(n)$. Consequently no most complex suffix-free language exists for $n \geqslant 4$.

The first four studies of most complex languages were done for the classes of regular languages [6], right ideals $[8,9,16]$, left ideals $[9,10,16]$, and two-sided ideals $[9,10,16]$. In those cases there exists a single witness over a minimal alphabet which, together with its permutational dialects, covers all the complexity measures. In the case of suffix-free languages such a witness does not exist. Our study is an example of a general problem: Given a class of regular languages, find the smallest set of witnesses over minimal alphabets that together cover all the measures. The witness of Definition 1 is conjectured to be over a minimal alphabet, unless the bound for product can be met by binary DFAs for every $n, m>c$, for some $c$; this is an 
Table 2: Suffix-free atom complexity continued.

\begin{tabular}{|c|c|c|c|c|}
\hline \hline$n$ & 6 & 7 & 8 & 9 \\
\hline \hline$|S|=0$ & $17 / 32 / 63$ & $33 / 64 / 127$ & $65 / 128 / 255$ & $129 / 256 / 511$ \\
\hline \hline$|S|=1$ & $33 / 81 / 187$ & $81 / 193 / 442$ & $193 / 449 / 1,017$ & $449 / 1,025 / 2,296$ \\
\hline$|S|=2$ & $\mathbf{5 3 / 1 5 6 / 4 0 6}$ & $156 / 427 / 1,086$ & $427 / 1,114 / 2,773$ & $1,114 / 2,809 / 6,859$ \\
\hline$|S|=3$ & $43 / \mathbf{1 6 6 / 5 0 1}$ & $\mathbf{1 6 6 / 5 4 2 / 1 , 5 4 8}$ & $\mathbf{5 4 2 / 1 , 6 1 1 / 4 , 4 2 5}$ & $1,611 / 4,517 / 12,043$ \\
\hline$|S|=4$ & $16 / 106 / 406$ & $106 / 462 / \mathbf{1}, \mathbf{5 4 8}$ & $462 / \mathbf{1}, \mathbf{6 4 6} / \mathbf{5}, \mathbf{0 8 3}$ & $\mathbf{1 , 6 4 6 / 5 , 2 4 5 / 1 5 , 3 6 1}$ \\
\hline$|S|=5$ & $* / 32 / 187$ & $32 / 249 / 1,086$ & $249 / 1,205 / 4,425$ & $1,205 / 4,643 / \mathbf{1 5}, \mathbf{3 6 1}$ \\
\hline$|S|=6$ & $* / 6 / 63$ & $* / 64 / 442$ & $64 / 568 / 2,773$ & $568 / 3,019 / 12,043$ \\
\hline$|S|=7$ & & $* / 7 / 127$ & $* / 128 / 1,017$ & $128 / 1,271 / 6,859$ \\
\hline$|S|=8$ & & & $* / 8 / 255$ & $* / 256 / 2,296$ \\
\hline$|S|=9$ & & & & $* / 9 / 511$ \\
\hline max & $53 / 166 / 501$ & $166 / 542 / 1,548$ & $542 / 1,646 / 5,083$ & $1,646 / 5,245 / 15,361$ \\
\hline ratio & $3.31 / 3.13 / 3.55$ & $3.13 / 3.27 / 3.09$ & $3.27 / 3.04 / 3.28$ & $3.04 / 3.19 / 3.02$ \\
\hline
\end{tabular}

open problem. The witness of Definition 2 is over a minimal alphabet, since five letters are required to meet the bound for syntactic complexity.

\section{Acknowledgments}

This work was supported by the Natural Sciences and Engineering Research Council of Canada (NSERC) grant No. OGP000087, and by the National Science Centre, Poland under grant number 2014/15/B/ST6/00615.

We are grateful to Sylvie Davies for careful proofreading, and for anonymous reviewers for their insightful comments.

\section{References}

[1] T. Ang, J.A. Brzozowski, Languages convex with respect to binary relations, and their closure properties, Acta Cybernet. 19 (2009) 445-464.

[2] J. Bell, J.A. Brzozowski, N. Moreira, R. Reis, Symmetric groups and quotient complexity of boolean operations, in: J. Esparza, et al. (Eds.), ICALP 2014, volume 8573 of LNCS, Springer, 2014, pp. 1-12.

[3] J. Berstel, D. Perrin, C. Reutenauer, Codes and Automata, Cambridge University Press, 2009. 
[4] J.A. Brzozowski, Complexity in convex languages, in: A. Dediu, H. Fernau, C. Martin-Vide (Eds.), LATA 2010, volume 6031 of LNCS, Springer, 2010, pp. $1-15$.

[5] J.A. Brzozowski, Quotient complexity of regular languages, J. Autom. Lang. Comb. 15 (2010) 71-89.

[6] J.A. Brzozowski, In search of the most complex regular languages, Int. J. Found. Comput. Sc. 24 (2013) 691-708.

[7] J.A. Brzozowski, Unrestricted state complexity of binary operations on regular languages, in: F.M. C. Câmpeanu, J. Shallit (Eds.), DCFS 2016, volume 9777 of LNCS, Springer Berlin / Heidelberg, 2016, pp. 60-72. For a revised version see http://arxiv.org/abs/1602.01387.

[8] J.A. Brzozowski, G. Davies, Most complex regular right ideals, in: H. Jürgensen, et al. (Eds.), DCFS, volume 8614 of LNCS, Springer, 2014, pp. 90-101.

[9] J.A. Brzozowski, S. Davies, Quotient complexities of atoms in regular ideal languages, Acta Cybernet. 22 (2015) 293-311.

[10] J.A. Brzozowski, S. Davies, B.Y.V. Liu, Most complex regular ideal languages, Discrete Math. Theor. Comput. Sci. (2016). To appear, also at http://arxiv . org/abs/1511.00157.

[11] J.A. Brzozowski, G. Jirásková, B. Li, J. Smith, Quotient complexity of bifix-, factor-, and subword-free regular languages, Acta Cybernet. 21 (2014) 507-527.

[12] J.A. Brzozowski, B. Li, Y. Ye, Syntactic complexity of prefix-, suffix-, bifix-, and factor-free regular languages, Theoret. Comput. Sci. 449 (2012) 37-53.

[13] J.A. Brzozowski, B. Liu, Quotient complexity of star-free languages, Int. J. Found. Comput. Sci. 23 (2012) 1261-1276.

[14] J.A. Brzozowski, C. Sinnamon, Complexity of left-ideal, suffix-closed, and suffixfree regular languages, 2016. http://arxiv.org/abs/1610.00728.

[15] J.A. Brzozowski, C. Sinnamon, Complexity of prefix-convex regular languages, 2016. http://arxiv.org/abs/1605.06697.

[16] J.A. Brzozowski, C. Sinnamon, Unrestricted state complexity of binary operations on regular and ideal languages, 2016. http://arxiv.org/abs/1602. 01387. 
[17] J.A. Brzozowski, C. Sinnamon, Complexity of right-ideal, prefix-closed, and prefix-free regular languages, Acta Cybernet. (2017). To appear. Also at http: //arxiv.org/abs/1605.06697.

[18] J.A. Brzozowski, M. Szykuła, Upper bounds on syntactic complexity of left and two-sided ideals, in: A.M. Shur, M.V. Volkov (Eds.), DLT 2014, volume 8633 of LNCS, Springer, 2014, pp. 13-24.

[19] J.A. Brzozowski, M. Szykuła, Complexity of suffix-free regular languages, in: A. Kosowski, I. Walukiewicz (Eds.), FCT 2015, volume 9210 of LNCS, Springer, 2015, pp. 146-159.

[20] J.A. Brzozowski, M. Szykuła, Upper bound for syntactic complexity of suffix-free languages, in: A. Okhotin, J. Shallit (Eds.), DCFS 2015, volume 9118 of LNCS, Springer, 2015, pp. 33-45. Full paper at http://arxiv.org/abs/1412.2281.

[21] J.A. Brzozowski, H. Tamm, Theory of átomata, Theoret. Comput. Sci. 539 (2014) 13-27.

[22] J.A. Brzozowski, Y. Ye, Syntactic complexity of ideal and closed languages, in: G. Mauri, A. Leporati (Eds.), DLT, volume 6795 of LNCS, Springer, 2011, pp. $117-128$.

[23] R. Cmorik, G. Jirásková, Basic operations on binary suffix-free languages, in: Z. Kotásek, et al. (Eds.), MEMICS, pp. 94-102.

[24] M. Crochemore, C. Hancart, Automata for pattern matching, in: G. Rozenberg, A. Salomaa (Eds.), Handbook of Formal Languages, volume 2, Springer, 1997, pp. 399-462.

[25] Y.S. Han, K. Salomaa, State complexity of union and intersection of finite languages, Int. J. Found. Comput. Sci. 19 (2008) 581-595.

[26] Y.S. Han, K. Salomaa, State complexity of basic operations on suffix-free regular languages, Theoret. Comput. Sci. 410 (2009) 2537-2548.

[27] M. Holzer, B. König, On deterministic finite automata and syntactic monoid size, Theoret. Comput. Sci. 327 (2004) 319-347.

[28] M. Holzer, M. Kutrib, Descriptional and computational complexity of finite automata - a survey, Information and Computation 209 (2011) 456 - 470. 
[29] S. Iván, Complexity of atoms, combinatorially, Inform. Process. Lett. 116 (2016) $356-360$.

[30] G. Jirásková, P. Olejár, State complexity of union and intersection of binary suffix-free languages, in: H. Bordihn, et al. (Eds.), NCMA, Austrian Computer Society, 2009, pp. 151-166.

[31] B. Krawetz, J. Lawrence, J. Shallit, State complexity and the monoid of transformations of a finite set, in: M. Domaratzki, A. Okhotin, K. Salomaa, S. Yu (Eds.), Proceedings of the Implementation and Application of Automata, (CIAA), volume 3317 of LNCS, Springer, 2005, pp. 213-224.

[32] E. Leiss, Succinct representation of regular languages by boolean automata, Theoret. Comput. Sci. 13 (2009) 323-330.

[33] A.N. Maslov, Estimates of the number of states of finite automata, Dokl. Akad. Nauk SSSR 194 (1970) 1266-1268 (Russian). English translation: Soviet Math. Dokl. 11 (1970), 1373-1375.

[34] J.E. Pin, Syntactic semigroups, in: Handbook of Formal Languages, vol. 1: Word, Language, Grammar, Springer, New York, NY, USA, 1997, pp. 679-746.

[35] G. Thierrin, Convex languages, in: M. Nivat (Ed.), Automata, Languages and Programming, North-Holland, 1973, pp. 481-492.

[36] S. Yu, State complexity of regular languages, J. Autom. Lang. Comb. 6 (2001) 221-234.

[37] S. Yu, Q. Zhuang, K. Salomaa, The state complexities of some basic operations on regular languages, Theoret. Comput. Sci. 125 (1994) 315-328.

\section{Appendix}

\section{Product of Suffix-Free Languages: Binary Case}

For $m \geqslant 6, n \geqslant 3$, let the first DFA be that of [23], except that the set of final states is changed to $\left\{2^{\prime}, 4^{\prime}\right\}$; thus let $\Sigma=\{a, b\}, \mathcal{D}_{m}^{\prime}(a, b)=\left(Q_{m}^{\prime}, \Sigma, \delta^{\prime}, 0^{\prime},\left\{2^{\prime}, 4^{\prime}\right\}\right)$, and let $\mathcal{D}_{n}(a, b)=\left(Q_{n}, \Sigma, \delta, 0,\{1\}\right)$, with the transitions shown in Figure 7 . Let $L_{m}^{\prime}(a, b)$ and $L_{n}(a, b)$ be the corresponding languages. 


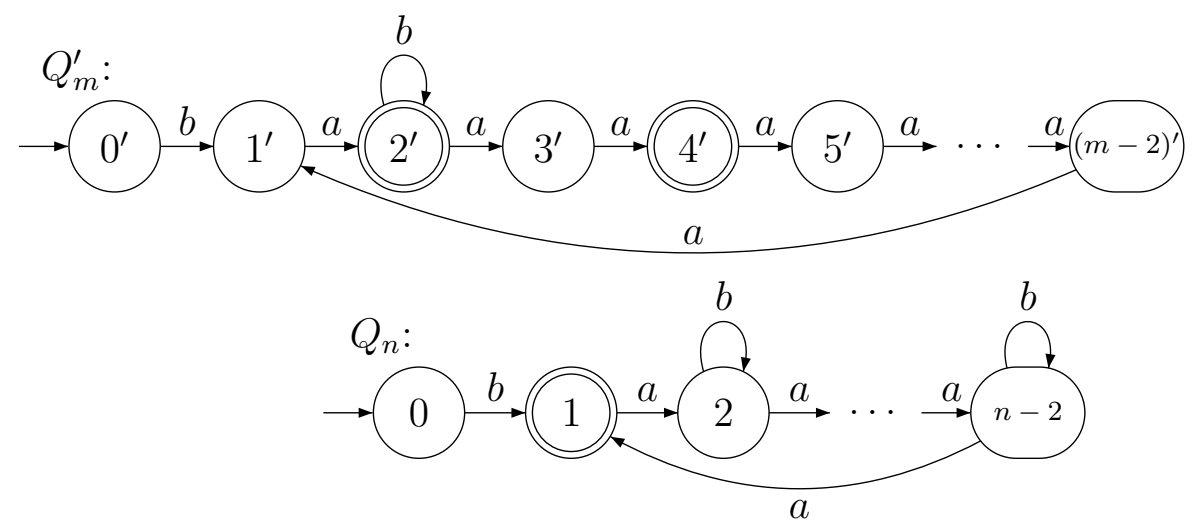

Figure 7: The witness DFA $\mathcal{D}_{m}^{\prime}$ and $\mathcal{D}_{n}$ for product. The transitions to the empty states $(m-1)^{\prime}$ and $n-1$ are omitted.

Theorem 13 (Product: Binary Case). For $m, n \geqslant 6, L_{m}^{\prime}(a, b)$ is suffix-free and $L_{m}^{\prime}(a, b) \cdot L_{n}(a, b)$ meets the bound $(m-1) 2^{n-2}+1$ when $m-2$ and $n-2$ are relatively prime.

Proof. First we need to show that $\mathcal{D}_{m}^{\prime}(a, b)$ is minimal and $L_{m}^{\prime}(a, b)$ is suffix-free. For minimality, it is easy to verify that every pair of states is distinguished by a word of the form $a^{i} b$. Suppose that $L_{m}^{\prime}(a, b)$ is not suffix-free; then there are some words $u, v$ such that $v \in L_{m}^{\prime}(a, b)$ and $u v \in L_{m}^{\prime}(a, b)$. Since there is no transformation mapping $0^{\prime}$ to itself, this means that $v$ maps both $0^{\prime}$ and $q^{\prime}=0^{\prime} u \in\left\{1^{\prime}, \ldots,(m-2)^{\prime}\right\}$ to a final state. Clearly $v=b w$ for some $w$ and so $q^{\prime}$ must be $2^{\prime}$. Then $w$ maps $1^{\prime}$ and $2^{\prime}$ to a final state. Since these two states cannot be focused to any state other than $(m-1)^{\prime}$, and $b$ sends every state from $\left\{1^{\prime}, \ldots,(m-2)^{\prime}\right\}$ except $2^{\prime}$ to $(m-1)^{\prime}$, we have $w=a^{i}$. But $a^{i}$ preserves the distance between the states in the cycle, and so $1^{\prime}$ and $2^{\prime}$ cannot be mapped simultaneously to $2^{\prime}$ and $4^{\prime}$. Minimality and suffix-freeness for $\mathcal{D}_{n}(a, b)$ has been shown in [23].

The proof for meeting the bound $(m-1) 2^{n-2}+1$ is similar to that of [23, Theorem 5]. We construct the NFA for $L_{m}^{\prime}(a, b) L_{n}(a, b)$ as usual. We show that $(m-1) 2^{n-2}+1$ subsets of the states are reachable and distinguishable. Since a subset $S$ without $n-1$ cannot be distinguished from $S \cup\{n-1\}$, we consider only $S$ as the representative for both $S$ and $S \cup\{n-1\}$. Similarly, a reachable subset $S$ contains 0 if and only if it contains either $2^{\prime}$ or $4^{\prime}$; we use only $S \backslash\{0\}$ as the representative for these subsets.

Let $X \subseteq\{1, \ldots, n-2\}$. If $X=\emptyset$, then clearly each of the $m-1$ subsets $\left\{p^{\prime}\right\}$ for $p \in\{1, \ldots, m-2\}$ is reachable by $b a^{p-1}$. Now let $X \neq \emptyset$. To show that each of the $(m-2)\left(2^{n-2}-1\right)$ subsets $S=\left\{p^{\prime}\right\} \cup X$ for $p \in\{1, \ldots, m-2\}$ is reachable, we follow the proof of [23, Theorem 5] exactly. In this proof note that $4^{\prime} \in F$ makes no difference, since $b$ maps $4^{\prime}$ to $(m-1)^{\prime}$, and so if applied to a subset containing $4^{\prime}$, it 
results in a set with $(m-1)^{\prime}$.

For the $2^{n-2}-1$ subsets $S=\left\{(m-1)^{\prime}\right\} \cup X$, suppose first $1 \notin X$; then $S$ is reachable from $\left\{1^{\prime}\right\} \cup X$ by $b$. If $1 \in X$, then $S$ is reachable from $\left\{4^{\prime}\right\} \cup X$ by $b$.

Finally, we show that all these $(m-1) 2^{n-2}$ sets together with the initial state $\left\{0^{\prime}\right\}$ are distinguishable. Set $\left\{0^{\prime}\right\}$ is distinguished from every other subset by $b a b$. Consider $\left\{p^{\prime}\right\} \cup X$ and $\left\{q^{\prime}\right\} \cup Y$ for distinct $p, q \in\{1, \ldots, m-2\}$, and some $X, Y \subseteq$ $\{1, \ldots, n-2\}$. Then $w=a^{m-p} b$ maps $p^{\prime}$ to $2^{\prime}$, and $q^{\prime}$ to $(m-1)^{\prime}$. Thus $\left(\left\{p^{\prime}\right\} \cup\right.$ $X) w$ contains the final state 1 , and $\left(\left\{q^{\prime}\right\} \cup X\right) w$ does not. Consider $\left\{p^{\prime}\right\} \cup X$ and $\left\{q^{\prime}\right\} \cup Y$ with $X \neq Y$; then $X$ and $Y$ differ in some $r \in\{1, \ldots, n-2\}$. Thus $a^{n-r-1}$ distinguishes these subsets. 